\title{
Sensorimotor Coding of Vermal Granule Neurons in the Developing Mammalian Cerebellum
}

\author{
ㅈelly H. Markwalter, ${ }^{1,2}{ }^{\circledR}$ Yue Yang, ${ }^{1}$ Timothy E. Holy, ${ }^{1}$ and ${ }^{\circledR A z a d ~ B o n n i}{ }^{1}$ \\ ${ }^{1}$ Department of Neuroscience, and ${ }^{2} \mathrm{MD}$-PhD Program, Washington University School of Medicine, St. Louis, Missouri 63110
}

The vermal cerebellum is a hub of sensorimotor integration critical for postural control and locomotion, but the nature and developmental organization of afferent information to this region have remained poorly understood in vivo. Here, we use in vivo two-photon calcium imaging of the vermal cerebellum in awake behaving male and female mice to record granule neuron responses to diverse sensorimotor cues targeting visual, auditory, somatosensory, and motor domains. Use of an activity-independent marker revealed that approximately half (54\%) of vermal granule neurons were activated during these recordings. A multikernel linear model distinguished the relative influences of external stimuli and co-occurring movements on neural responses, indicating that, among the subset of activated granule neurons, locomotion $(44 \%-56 \%)$ and facial air puffs $(50 \%)$ were more commonly and reliably encoded than visual $(31 \%-32 \%)$ and auditory (19\%-28\%) stimuli. Strikingly, we also uncover populations of granule neurons that respond differentially to voluntary and forced locomotion, whereas other granule neurons in the same region respond similarly to locomotion in both conditions. Finally, by combining two-photon calcium imaging with birth date labeling of granule neurons via in vivo electroporation, we find that early-and late-born granule neurons convey similarly diverse sensorimotor information to spatially distinct regions of the molecular layer. Collectively, our findings elucidate the nature and developmental organization of sensorimotor information in vermal granule neurons of the developing mammalian brain.

Key words: calcium imaging; cerebellar development; cerebellar granule neuron; sensorimotor encoding; two-photon imaging

\section{Significance Statement}

Cerebellar granule neurons comprise over half the neurons in the brain, and their coding properties have been the subject of theoretical and experimental interest for over a half-century. In this study, we directly test long-held theories about encoding of sensorimotor stimuli in the cerebellum and compare the in vivo coding properties of early- and late-born granule neurons. Strikingly, we identify populations of granule neurons that differentially encode voluntary and forced locomotion and find that, although the birth order of granule neurons specifies the positioning of their parallel fiber axons, both early-and late-born granule neurons convey a functionally diverse sensorimotor code. These findings constitute important conceptual advances in understanding the principles underlying cerebellar circuit development and function.

\section{Introduction}

The cerebellum is a key center of sensorimotor processing in the brain. By integrating diverse neural inputs that relay information

Received Jan. 11, 2019; revised May 18, 2019; accepted June 18, 2019.

Author contributions: K.H.M., T.E.H., and A.B. designed research; K.H.M. performed research; K.H.M., Y.Y., and T.E.H. analyzed data; K.H.M. wrote the first draft of the paper; Y.Y. edited the paper; T.E.H. and A.B. wrote the paper.

This work was supported by National Institutes of Health Grant NS041021 to A.B. and Grant NS068409 to T.E.H., the Mathers Foundation to A.B., and National Institutes of Health Medical Scientist Training Program Grant T32 GM07200 to K.H.M. We thank the machine shop at Washington University and the Washington University Center for Cellular Imaging for technical support; and Martha Bagnall, Pablo Blazquez, Ed Han, and members of the Bonni and Holy laboratories for helpful discussions and critical review of the manuscript.

The authors declare no competing financial interests.

Correspondence should be addressed to Azad Bonni at bonni@wustl.edu or Timothy E. Holy at holy@wustl.edu. Y. Yang's present address: Department of Neurobiology, Northwestern University, Evanston, IL 60208.

https://doi.org/10.1523/JNEUROSCI.0086-19.2019

Copyright $\odot 2019$ the authors about internal and external contexts, the cerebellum facilitates motor coordination, adaptation, and learning. However, the function and organization of cerebellar inputs that enable these tasks have remained poorly understood.

Within the cerebellum, the vermal region is thought to play a key role in locomotor coordination and postural control (Holmes, 1922; Shik and Orlovsky, 1976; Armstrong, 1986; Bastian et al., 1998; Thach and Bastian, 2004; Vinueza Veloz et al., 2015). Accordingly, the vermis receives dense mossy fiber inputs from spinocerebellar tracts encoding limb muscle activity and joint position (Arshavsky et al., 1972; Matsushita et al., 1979; Matsushita and Ikeda, 1980; Armstrong, 1988; Päällysaho et al., 1991; Orlovsky et al., 1999; Bosco et al., 2005) as well as from premotor and motor regions of the cerebral cortex that arrive via the pontine nuclei (Kelly and Strick, 2003; Coffman et al., 2011). How- 
ever, the vermis also receives mossy fiber inputs from regions related to visual, auditory, tactile, and vestibular processing as well as inputs relayed from cortical association areas (Snider and Stowell, 1944; Aitkin and Boyd, 1975; Huang et al., 1982; Wiesendanger and Wiesendanger, 1982; Stein and Glickstein, 1992; Glickstein, 1997). This broad convergence of information has been hypothesized to endow the vermis with a role in sensorimotor integration and associative learning (Stein and Glickstein, 1992; Sawtell, 2010; Huang et al., 2013; Ishikawa et al., 2015; Giovannucci et al., 2017), but how the afferent information is represented and processed within the cerebellum to facilitate such roles in vivo remains unclear.

The organization of information arriving via the mossy fibergranule neuron system has historically been underexplored due to the technical challenges of recording large populations of granule neurons in vivo. Recent studies have overcome these challenges and begun to offer new insights into the diversity, density, and dynamic nature of the information encoded by granule neurons (Gilmer and Person, 2017; Giovannucci et al., 2017; Knogler et al., 2017, 2019, Wagner et al., 2017, 2019; Yamada et al., 2019). However, the response properties of mammalian granule neurons to a battery of untrained sensorimotor stimuli have not yet been systematically explored. Furthermore, the developmental programs that govern granule neuron sensorimotor coding remain unclear. Intriguingly, the birth order of granule neurons appears to influence parallel fiber positioning (Espinosa and Luo, 2008), suggesting that coding properties of early- versus lateborn granule neurons could influence information processing in the cerebellar cortex. However, the influence of birth order on organization of sensorimotor information arriving to granule neurons remained unknown.

To better understand the nature and developmental organization of sensorimotor information encoded by granule neurons in the mammalian cerebellar vermis, we have imaged $>1000$ individual granule neurons in lobule VIA, an optically accessible vermal region associated with multiple sensorimotor contexts (Stein and Glickstein, 1992; Stoodley and Schmahmann, 2009; Yang et al., 2016; Giovannucci et al., 2017; Wagner et al., 2017; Yamada et al., 2019), during locomotion and upon exposure to visual, auditory, and somatosensory cues. We have also developed a multikernel linear model that disambiguated granule neuron responses to sensorimotor stimuli from responses to cooccurring movements. This approach revealed granule neurons with diverse temporal and combinatorial response properties. Strikingly, populations of granule neurons responded differentially to voluntary versus forced locomotion. In addition, combining in vivo two-photon calcium imaging with in vivo birth date-dependent labeling of granule neurons in awake behaving juvenile mice revealed that, while the birth order of granule neurons profoundly influences the positioning of their parallel fiber axons in the molecular layer (ML), both early- and late-born granule neurons respond to the panoply of sensorimotor stimuli. Together, our findings uncover properties of vermal granule neuron responses to diverse sensorimotor stimuli and reveal that this sensorimotor code is conveyed broadly to distinct molecular layer regions of the mammalian cerebellar cortex.

\section{Materials and Methods}

\section{Animals}

For in vivo imaging experiments, we crossed mice expressing the calcium indicator GCaMP6f in a Cre-dependent fashion (Ai95, The Jackson Laboratory) with $G A B A(A) R \alpha 6$-Cre driver mice (Fünfschilling and Reichardt, 2002). Due to male germline expression of the Cre recombinase, female $G A B A(A) R \alpha 6$-Cre animals were used for all matings. WT C57BL/6 mice of either sex were used for histologic experiments characterizing positioning of electroporated granule neurons. All experiments were done according to protocols approved by the Animal Studies Committee of Washington University School of Medicine and in accordance with National Institutes of Health guidelines. Male and female mice were used for all experiments, and mice were purchased and maintained under pathogen-free conditions.

\section{In vivo electroporation}

In vivo electroporation of postnatal mouse pups was done as described previously (Huynh et al., 2011; Yamada et al., 2014; Yang et al., 2016) with modifications. For injection of pups older than P10, a small $(\sim 5$ $\mathrm{mm}$ ) incision was made in the dorsal scalp of lightly anesthetized mouse pups (isoflurane: $5 \%$ in $\mathrm{O}_{2}$ for induction, $1 \%-2 \%$ for maintenance), and the occipital bone overlying the cerebellum was targeted for midline injection. In younger pups, no incision was necessary for accurate targeting of the cerebellum. In all cases, $3 \mu \mathrm{g}$ of the DNA plasmid ( $\mathrm{pCAG}-\mathrm{GFP}$ or pCAG-mCherry, prepared at $1.5 \mu \mathrm{g} / \mu \mathrm{l}$ ) was injected, and pups were subjected to four $50 \mathrm{~ms}$ electrical pulses of $120 \mathrm{mV}$ at $950 \mathrm{~ms}$ intervals. Incisions were closed with suture, and electroporated pups were returned to dams until weaning age.

\section{Histologic procedures}

Immunohistochemical analyses were performed as described previously (Yamada et al., 2014; Valnegri et al., 2017). For characterization of GCaMP6f expression, brains of 4- to 5-week-old mice were perfused transcardially with $4 \%$ PFA and $4 \%$ sucrose, postfixed overnight, and stored in $30 \%$ sucrose solution. For morphologic quantification of electroporated neurons, the electroporated cerebellum was collected at postnatal day 22 (P22) and fixed in 4\% PFA and 4\% sucrose. In all cases, brains were frozen, sectioned sagittally at $30 \mu \mathrm{m}$, and stained with antibodies against calbindin (Millipore AB1788, 1:250), GFP (Abcam ab13970, 1:250), or DsRed (Clontech 632496, 1:250), as well as the DNA dye bisbenzimide (Hoechst). Images of labeled sections were taken on an AxioScan Z1 fluorescence imaging system (Carl Zeiss) or an Olympus FV1200 confocal system.

\section{Cranial window implant surgical procedures}

For imaging experiments, a 3-mm-diameter circular craniotomy was made overlying the dorsal cerebellum of mice on P26-P28. Mice were anesthetized with isoflurane ( $5 \%$ induction, $1 \%-2 \%$ maintenance) during surgery. Dexamethasone (2 mg/kg i.m.), carprofen (5 mg/kg s.c.), and buprenorphine $(0.5-1.0 \mathrm{mg} / \mathrm{kg}$ s.c.) were administered to reduce brain swelling and inflammation and to provide analgesia. The craniotomy overlapped portions of the occipital and interparietal bones; it generally extended the full width of the vermis, exposing the dorsal surface of lobule VI as well as parts of lobules IV/V and VII. The cranial window was installed using cyanoacrylate followed by dental cement (Ortho-Jet, Lang) to secure a double-layered glass coverslip over the exposed salinecovered brain. The two coverslip layers were joined by UV-cured optical adhesive (Norland Products, NOA71) and positioned such that the lower layer ( $3 \mathrm{~mm}$ diameter, $0.15 \mathrm{~mm}$ thick) fit snugly inside the craniotomy and the upper layer ( $5 \mathrm{~mm}$ diameter, $0.15 \mathrm{~mm}$ thick) contacted the intact skull. A titanium headplate for head fixation was centered over the craniotomy and secured using dental cement mixed with carbon (484164, Sigma-Aldrich) to help reduce light transmission through the skull. Mice were imaged at least $5 \mathrm{~d}$ after surgery.

\section{Two-photon microscopy}

Dual-color imaging was performed on a Bruker multiphoton system equipped with a Ti:Sapphire laser (MaiTai eHP DeepSee, SpectraPhysics), a $20 \times$ water-immersion objective (NA 1.0, Olympus), and $\mathrm{GaAsP}$ detector elements. All images were acquired at $20 \mathrm{~Hz}(\sim 2 \mu$ s dwell time per pixel) with the excitation laser tuned to $920 \mathrm{~nm}$. Signals from GCaMP6f and mCherry fluorescent indicators were collected simultaneously and separated based on their emission spectra.

Prairie View software (Bruker) was used to synchronize image acquisition, stimulus triggers, and recordings of treadmill velocity. The LED and speaker were triggered directly, the treadmill motor was controlled 
Table 1. Parameters and timing of sensorimotor stimuli

\begin{tabular}{|c|c|c|c|}
\hline Stimulus & Parameters & Timing per block ${ }^{a}$ & Hardware \\
\hline Air puff & $\begin{array}{l}\text { Facial: } 20 \text { psi, targeting right periorbital area, calibrated } \\
\text { to cause an eyeblink } \\
\text { Trunk: } 20 \text { psi, targeting right lateral trunk, with some } \\
\text { variation introduced by mouse trunk movements }\end{array}$ & Three 300 ms air puffs with 2000 ms ISI & Ingersoll Rand AR0 37121-600 Air Regulator \\
\hline Tone & $\begin{array}{l}\text { Beep: steady tone at } \sim 90 \mathrm{~dB} \\
\text { Warble: tone } \sim 90 \mathrm{~dB} \text { with frequency alternating }\end{array}$ & Three 1000 ms beeps with 1000 ms ISI & Speaker: SparkFun, COM-13939, setting 1, driven at 5 V \\
\hline & between $2800 \mathrm{~Hz}$ and $3200 \mathrm{~Hz}$ & 5000 ms tone-warble with $50 \%$ duty cycle at rate of $1 \mathrm{~Hz}$ & Speaker: SparkFun, COM-13939, setting 8, driven at $5 \mathrm{~V}$ \\
\hline Light & $\begin{array}{l}\text { Stable: blue LED }(\sim 465 \mathrm{~nm}) \\
\text { Flash: blue LED }(\sim 465 \mathrm{~nm}) \text { alternating on and off }\end{array}$ & $\begin{array}{l}5000 \text { ms LED on } \\
5000 \text { ms LED flashing with } 50 \% \text { duty cycle at rate of } 5 \mathrm{~Hz}\end{array}$ & LED: SparkFun, COM-00529 \\
\hline Treadmill & $\begin{array}{l}\text { Fast: } 10 \text { rpm continuous } \\
\text { Slow: } 5 \text { rpm continuous }\end{array}$ & 5000 ms treadmill-on & Stepper Motor: SparkFun ROB-09238 \\
\hline Nothing & Imaging without any external stimulus presentations & 9 s block & None \\
\hline
\end{tabular}

${ }^{a}$ Each stimulus block was $9 \mathrm{~s}$ total. Each block began and ended with a no-stimulus period of $1.9-2.2 \mathrm{~s}$ duration. The intervening stimulus-on periods were as described per condition. ISI, Interstimulus interval.

through an Arduino Due microcontroller, and the air puffs were triggered through an external stimulus delivery system (Automate Scientific, ValveLink 8.2).

For all imaging studies, mice were head-fixed on a custom-built motorized treadmill (adapted from Heiney et al., 2014) that was suspended on low-friction ball bearings and was freely rotatable, except during forced treadmill epochs. At the time of imaging (P32-P34), surface vasculature was inspected and used to identify the boundaries of lobule VI. We selected imaging regions within the anterior half of lobule VI, approximately corresponding to lobule VIA, that included several mCherry-positive granule cells labeled by in vivo electroporation. Imaging fields were generally located within the top $150 \mu \mathrm{m}$ of the internal granule layer (IGL), covered an average surface area of $10,923 \mu \mathrm{m}^{2}$, were preferentially aligned with their long axis in an anteroposterior direction, and extended an average of $63 \mu \mathrm{m}$ in the mediolateral direction (range $28-112 \mu \mathrm{m})$. Individual FOVs were therefore likely to fall either within one module or between two adjacent modules as defined by mossy fiber terminals, which cluster in $\sim 100$ - to $150-\mu \mathrm{m}$-wide parasagittally oriented stripes (Ji and Hawkes, 1994; Gebre et al., 2012; Huang et al., 2013; Apps et al., 2018). Two to seven FOVs were imaged per animal, suggesting that the composite dataset includes granule neurons sampled from multiple modules.

Imaging and behavioral data were collected in stimulus blocks of $9 \mathrm{~s}$ duration. Blocks were presented in a pseudorandomized order with a randomized interblock interval of 1-25 s. Imaging data for each FOV, including three trials of each stimulus block, were acquired within 10-15 min, and 2-7 FOVs were imaged per animal. Total imaging time for each mouse did not exceed $120 \mathrm{~min}$.

For forced treadmill stimulus blocks, fixed-speed treadmill rotation was driven by a stepper motor (SparkFun Electronics, ROB-09238) at 4 or $8 \mathrm{~cm} / \mathrm{s}$. These speeds were selected based on visual inspection of untrained behavior of juvenile mice walking on the rotating treadmill. Specifically, the speeds were chosen to be relatively slow and fast speeds at which the mice were nonetheless able to keep pace and demonstrate a continuous gait cycle for a $5 \mathrm{~s}$ trial. During forced as well as voluntary epochs, treadmill rotations were recorded by a rotary encoder (SparkFun Electronics, COM-11102). No video recordings were collected during data acquisition. Timing and parameter specifics of the stimulus blocks are summarized in Table 1 .

\section{Experimental design and statistical analysis}

Histologic analysis. To quantify the position of electroporated parallel fibers within the molecular layer, we first used the calbindin-immunoreactive signal to delineate the extent of the molecular layer. Line segments oriented perpendicular to the pial surface were drawn from the pia to the top of the Purkinje cell somas at 100-200 $\mu \mathrm{m}$ intervals along the dorsal cerebellar surface. These lines were drawn while blinded to the age of electroporation and fluorescence channel of electroporated neurons. ML thickness was quantified as mean line length per lobule.

Next, the intensity profile of electroporated fluorescence signal along each previously drawn line was quantified, and the relative location of the peak signal along the line was determined. To avoid artifact due to local fluorescence hotspots or intersection of occasional labeled Bergmann glial cell, images were smoothed before intensity profiling, and lines intersecting labeled glia were excluded from analysis. Smoothing was accomplished using two applications of the ImageJ Smooth function, which replaces each pixel with the average of its $3 \times 3$ neighborhood.

To quantify the distribution of labeled granule neuron somas within the IGL, the Hoechst signal was used to identify the extent of the IGL and divide it into thirds. The electroporated fluorescence channel was then examined, and the signal intensity per unit area was quantified. IGL delineations were done blinded to electroporated signal and age at electroporation.

Calcium imaging data analysis. Image analysis was performed using custom ImageJ, MATLAB, and Julia scripts. Image registration was accomplished using nonrigid motion correction based on template matching as previously described (Pnevmatikakis and Giovannucci, 2017). We calculated shifts from our sparse-labeled structural channel (mCherry) and applied the displacements to both the structural and functional (GCaMP6f) channels. Image registration successfully resolved withinplane motion. To ensure that out-of-plane motion did not contaminate the analyzed calcium transients, we used a two-step quality control process. First, taking advantage of the presence of an activity-independent label, we examined the time course of mCherry signal from the sparsely labeled somas. As expected, mCherry signals remained stable across imaging. On rare occasions when the signal of any mCherry soma in a FOV varied beyond 3 SDs from its running mean, the entire frame was excluded from analysis. These dropped-frame events were rare, accounting for $<1 \%$ of all frames. If a single trial ever had $>5 \%$ of frames excluded, the entire trial was discarded; this was also a rare occurrence $(<2 \%$ of all trials). Second, we used a standard approach of analyzing the statistics of the GCaMP6f signal to identify statistically significant positive-going transients with $<5 \%$ false-positive error rate of being attributable to motion (Dombeck and Tank, 2014). Only the trials and transients passing both the mCherry and GCaMP6f-based evaluations were analyzed further, thus providing high confidence that the analyzed calcium activity was minimally contaminated by motion artifact.

To identify ROIs, we developed and used a custom cell segmentation algorithm optimized for separation of signal sources in densely packed regions, such as the IGL. Briefly, our method uses a non-negative matrix factorization approach similar to those described previously (Maruyama et al., 2014; Pnevmatikakis et al., 2016) to separate signals based on their patterns of spatial and temporal coherence. The method can attribute signal from a single pixel to multiple ROIs, provided that the contributing ROIs have some epochs of nonsynchronous activity. By allowing separation of signal from overlapping ROIs, this approach reduces contamination by adjacent signal sources. In addition, this approach removes any diffuse time-variant signal before segmenting cells, thus accounting for a diffuse calcium signal that may originate from neuropil composed of granule cell ascending axons.

After segmentation, all identified ROIs were manually rated on two criteria. First, with reference to the mean GCaMP6f image and a sliding average of the entire time series, each ROI was inspected and rated as 
unambiguously nuclear-cleared or not. Absence of nuclear clearing is often used as an indicator of poor neuronal health, particularly when overexpression of the calcium indicator is a concern (Tian et al., 2009). In our case, with stable transgenic expression of the GCaMP6f protein and $a$ priori concern for a nonsomatic source of calcium signal associated with granule neuron dendritic claws (Wagner et al., 2017), we instead used the criterion of nuclear clearing as a mechanism to reduce contamination by nonsomatic structures (Wagner et al., 2017). Validation against the sparse mCherry label confirmed that ROIs located at cerebellar glomeruli lacked nuclear clearing, but we cannot rule out the possibility that a small minority of our verified nuclear-cleared ROIs also corresponded to glomeruli.

Second, in separate analyses, we evaluated the overlay of each ROI's spatial profile with the mean mCherry signal and used the sparse label to rate each ROI as somatic, glomerular, or not sparsely labeled. Importantly, all ratings were done blinded to the opposite channel signal, to the calcium signal of the ROI being rated, and to the electroporation age of the animal. All detected, nuclear-cleared ROIs were used in analyses of sensorimotor coding of granule neuron somas (see Figs. 3-6). All detected ROIs corresponding to sparsely labeled somas were used in analyses of the effects of birth order on functional coding (see Fig. 8).

For each segmented ROI, we calculated a modified $z$ score as follows:

$$
Z=\frac{0.67 *\left(X-X_{25 \text { th percentile }}\right)}{\text { Median absolute deviation of } X \text { from } X_{25 \text { th percentile }}}
$$

The 25th percentile of the calcium trace, rather than the median, was used as the baseline for the modified $z$ score calculation because visual inspection revealed this value to better reflect the signal baseline. The modified $z$ scores in epochs of statistically significant transients were used for all subsequent analyses.

Locomotion analysis. Rotary encoder data were analyzed offline to calculate movement velocities. Briefly, the quadrature-encoded gray code was converted to revolutions per minute and, accounting for treadmill diameter, to a linear velocity. Encoder data were collected at $10 \mathrm{kHz}$.

For cumulative distribution plots summarizing frequency of motor behaviors in each animal (see Fig. $3 B$ ), we analyzed encoder data from all time points when the treadmill was under voluntary control. These data came from multiple trials and multiple FOVs per animal. The foam treadmill required very little force to rotate, and the encoder was capable of detecting movements as small as $0.05 \mathrm{~cm}$ at the surface of the treadmill. Consequently, small postural adjustments as well as bouts of locomotion were detected. To provide an initial description of the nature as well as the frequency of mouse behaviors detected, any frame in which velocity was non- 0 was counted as movement and any frame in which velocity surpassed $3 \mathrm{~cm} / \mathrm{s}$ was counted as locomotion (see Fig. $3 B$ ). For each animal, we calculated the mean speed during movement-containing or locomotion-containing frames across all recorded trials and FOVs.

Individual mice engaged in differing amounts of voluntary locomotion during each recording (see Fig. 3B). To avoid drawing conclusions about neural responses to locomotion based on a limited sample, the recordings containing $<2.5 \mathrm{~s}$ of locomotion (total of 11) were excluded from locomotion analyses (see Fig. $3 D, E$ ). The cutoff of $2.5 \mathrm{~s}$ is comparable with the minimum amount of stimulus-on time used in analyses of responsivity to the other external stimuli. In total, locomotor responses were analyzed for 1196 granule neurons recorded from 71 FOVs (Fig. $3 D, E)$. Responsivity indices were calculated as the conditional probability that a frame exhibited a statistically significant calcium transient given that it met the movement criterion of interest as follows:

Free wheel responsivity index

$=\frac{\text { Number of Free Wheel Movement Frames With Calcium Transient Occurring }}{}$ Number of Free Wheel Movement Frames

Motorized wheel responsivity index

Number of Motorized Treadmill Frames With Calcium Transient Occurring Number of Motorized Treadmill Frames

Because this approach does not normalize for the cell's firing rate during stimulus-off epochs, it is informative only in cell populations with low basal firing rates, such as cerebellar granule neurons.
The responsivity indices plotted in Figure $4 E$ were calculated by the same method as above. Figure $4 E$ displays the subset of cells from Figure $3 D$ that meet inclusion criteria for the multikernel linear model analysis (i.e., those with three trials per stimulus), and each datapoint is displayed with a color code reflecting its responsivity as determined by the multikernel linear model analysis described below.

Multikernel linear model. We used a multikernel linear model based on principles that have been described previously (Simoncelli et al., 2004; Dayan and Abbott, 2005). This approach is a type of multiple linear regression. Solving for kernels rather than constant coefficients that describe the relationships between the stimuli and neural activity allows for time-dependent stimulus-response relationships to be identified. Briefly, given a set of $i$ time-dependent stimuli $S_{t, i}$ including external perturbations and recorded behavioral information (i.e., movement velocity), and a time-dependent response vector $r_{t}$, we found the set of kernel vectors, one per stimulus, that when convolved with the stimuli and summed would best reproduce the response as follows:

$$
\bar{r}_{t}=\sum_{\tau, i} S_{t-\tau, i} k_{\tau, i}
$$

by minimizing the sum of square errors as follows:

$$
\Sigma=\frac{1}{2} \sum_{t}\left(r_{t}-\bar{r}_{t}\right)^{2}
$$

For most stimuli, the kernel was allowed a duration of 40 frames, thus allowing $2 \mathrm{~s}$ of stimulus history to influence the response at time $t$. As an exception, for locomotor-related stimuli (voluntary movement, treadmill fast, and treadmill slow), the kernels had only 10 time points (500 $\mathrm{ms}$ ) of history. Use of the shorter kernel allowed retention of more data during the $\sim 2$ s baseline period of each trial (i.e., before stimulus presentations), even though that period lacked a full $2 \mathrm{~s}$ history of voluntary movement behavioral data. This choice was made in accordance with visual inspection of neural activity, which revealed minimal persistence of calcium signal, beyond that predicted by GCaMP6f decay kinetics, after bouts of locomotion.

Given the binary nature of the treadmill stimulus signal, in contrast to the temporally diverse responses it evoked, we added two additional stimuli to the model that were non- 0 at moments of treadmill initiation or termination, respectively. The addition of these stimuli improved the model's prediction of responses to the treadmill stimulus and were therefore used for all analyses. For each cell, kernels were solved for all three treadmill-associated features (initiation, steady-state, and termination), and the cell's net characteristic response to the treadmill (see Fig. 5E) was defined as the sum of the three characteristic responses.

As neural activity in the cerebellum can precede movement, we evaluated whether the model's accuracy was improved when the voluntary movement kernel was allowed to consider treadmill velocity in the near future $(250,500$, or $1000 \mathrm{~ms}$ ) and/or more distant past (up to $2000 \mathrm{~ms}$ ). This additional information did not dramatically improve the model's performance and was therefore not incorporated into the analyses presented. The lack of predictive improvement should not be interpreted to mean that granule neuron activity does not precede some aspects of locomotor behavior. Rather, it likely reflects the comparative simplicity of mapping neural activity to locomotor velocity as opposed to mapping it to all features and dimensions of mammalian locomotion.

To enforce a match only in places where complete information was known, we implemented a weighting vector $\omega_{t}$, where

$$
\omega_{t}=\left\{\begin{array}{c}
1 \text { if } r_{t} \text { known and could be predicted from } S \text { and } k \\
0 \text { otherwise }
\end{array}\right.
$$

such that

$$
\Sigma=\frac{1}{2} \sum_{t} \omega_{t}\left(r_{t}-\bar{r}_{t}\right)^{2}
$$

At times where $\omega_{t}=0$, the predicted value was allowed to be arbitrary. For example, $\omega_{t}$ always equaled 0 during the first $500 \mathrm{~ms}$ of each trial, as 
stimulus history was unknown for the voluntary movement stimulus occurring during that period.

Other than minimizing $\Sigma$, no additional constraints were applied to the kernels. To ensure that interpretations were not confounded by potential degeneracies in the solutions, we restricted our analysis and interpretation to the convolution of each kernel with the time course of its corresponding stimulus. The resulting "reconstructed responses" (see Fig. $4 A$ ) represented the neural activity predicted to have occurred in association with each stimulus individually. In other analyses, a single "characteristic response" was calculated as the convolution of each kernel with the time course of a single presentation of the relevant stimulus. Characteristic responses could be directly compared to evaluate the variety of neural responses associated with each stimulus (see Fig. 5C,E). The characteristic responses solved for the forced locomotion stimulus, as well as those for any other stimulus defined by a constant value that lasts longer than the $2 \mathrm{~s}$ kernel duration, will by definition have a period of constant value. This explains the constant values lasting from $2 \mathrm{~s}$ after treadmill initiation until treadmill termination in the characteristic responses to forced locomotion (see Fig. 5E).

To assess the accuracy of the model, we computed power spectra for the recorded neural activity and for the residual, that is, the difference between the recorded activity and the response predicted by the model. Accuracy was reported at each frequency as the percentage of power in the recorded neural signal that was present in the residual.

For the schematic of the multikernel model shown in Figure 4A, Stimulus 1 was defined to induce firing at $50 \mathrm{~Hz}$ and Stimulus 2 was defined to induce a burst of firing every $250 \mathrm{~ms}$, with $100 \mathrm{~Hz}$ intraburst frequency. The corresponding calcium activity was determined by convolving the firing pattern with a GCaMP6f kernel modeled as an exponential decay with $400 \mathrm{~ms}$ half-life (Chen et al., 2013). As in the real dataset, the model was solved using $2 \mathrm{~s}$ of stimulus history to solve for the kernels that define a reconstructed response optimally fitted to the original calcium activity.

Analysis of stimulus responsivity. To assess stimulus responsivity, we first regressed out the neural activity attributed to voluntary movements and then analyzed whether the remaining level of neural activity during each peristimulus period of interest was significantly higher than the activity during no-stimulus periods. This analysis was done using a statistical scramble approach (Peters et al., 2014). Briefly, the neural activity during each peristimulus epoch, defined as the moment of stimulus initiation through $1 \mathrm{~s}$ after stimulus termination, was extracted as a contiguous segment. The activity during each of the many no-stimulus epochs was extracted likewise. These segments were concatenated in 1000 randomly shuffled orders to form composite traces, and the mean during the true peristimulus period was compared with the mean during an equivalent period of time in each shuffled trace. If the true mean was $>99 \%$ of shuffled means, the neuron was considered to be responsive to that stimulus trial. For populational response statistics (see Fig. $5 \mathrm{~A}$ ), neurons were counted as responsive if they were significantly responsive to at least one of three trials, and neurons without three trials of a particular stimulus were excluded from the responsivity analysis of that stimulus.

Responsivity to voluntary movement was assessed by an analogous method. The neural activity attributed to other stimuli by the multikernel linear model was regressed out, and the remaining unexplained activity was analyzed to determine whether it was higher during the true peristimulus periods for voluntary movement compared with nomovement periods. The peristimulus periods were defined as all frames within $250 \mathrm{~ms}$ of a frame with voluntary movement velocity $>3 \mathrm{~cm} / \mathrm{s}$. Bouts of treadmill movement without velocity $>3 \mathrm{~cm} / \mathrm{s}$ were excluded from the analysis to preferentially analyze locomotion rather than small postural adjustments or isolated limb movements. In addition, as described above for the initial locomotor-responsivity analyses, neurons without at least $2.5 \mathrm{~s}$ of recordings when voluntary movement was $>3 \mathrm{~cm} / \mathrm{s}$ were excluded from this analysis due to the limited sample duration.

For visualization of neural response diversity, the cell/stimulusspecific kernels were convolved with the stimulus time courses to evaluate the dynamics of each neuron's typical response. Only neurons that responded to at least two of the three trials were visualized. Responses to the air puff stimulus were sorted based on FWHM (see Fig. 5C). Re- sponses to the fast treadmill were sorted using $k$-means clustering $(k=3)$ because sorting by a single feature did not effectively illustrate the response variability. $k=3$ was chosen because the characteristic responses were determined as a composite of the responses to three treadmill features (initiation, steady-state, and termination) (see Fig. 5E).

To calculate the dual responsivity heatmap in Figure $6 C$, we considered each pair of two stimuli and calculated the observed probability that a neuron within the subpopulation of neurons responsive to Stimulus A also responded to Stimulus $\mathrm{B}$, that is, $\mathrm{P}(\mathrm{B} \mid \mathrm{A})$. This observed probability was compared with the expected probability that a neuron taken from the total population responded to Stimulus $\mathrm{B}$, that is, $\mathrm{P}(\mathrm{B})$ (see Fig. $5 \mathrm{~A}$ ). These two ratios were compared to identify whether granule neurons responded to certain pairs of stimuli more or less frequently than expected by chance. In other words, for each pair of Stimuli $A$ and B, we tested the null hypothesis $\mathrm{P}(\mathrm{B} \mid \mathrm{A})=\mathrm{P}(\mathrm{B})$, which is true if $\mathrm{A}$ and $\mathrm{B}$ are independently assorted events and is false if responsivity of a neuron to stimulus A affects the likelihood that it responds to stimulus B.

Linear discriminant analysis. We performed a linear discriminant analysis as described previously (Campbell et al., 2008) to determine whether the neural responsivities determined by the kernel analysis could be used to reliably distinguish populations of granule neurons. Each neuron's functional response was summarized as a nine-element binary vector describing whether or not the neuron was responsive to each of the nine stimuli: air puff to face, air puff to trunk, light flashing, light sustained, tone beep, tone warble, forced locomotion fast, forced locomotion slow, and free locomotion (Table 1). The linear discriminant analysis identified the weighted combination of these nine stimuli that could best sort the neurons into predefined classes (i.e., early-born vs late-born). As a positive control, the responsivities derived from the multikernel linear model were used to distinguish two populations of neurons defined based on the independent movement responsivity analyses as preferentially responsive to free locomotion (free-wheel responsivity index $>0.6$ and motorized-wheel responsivity index $<0.3$ ) or preferentially responsive to forced locomotion (free-wheel responsivity index $<0.3$, motorized-wheel responsivity index $>0.6$ ).

\section{Code availability}

The code needed to implement the multikernel linear model is publicly available on GitHub (https://github.com/HolyLab/LinearKernel Models.jl).

\section{Results}

\section{Calcium imaging of bona fide granule neuron somatic} responses to sensorimotor stimuli in awake behaving mice

To uncover coding properties in the developing cerebellar cortex, we subjected awake behaving juvenile mice that expressed the calcium indicator GCaMP6f in granule neurons to two-photon calcium imaging (Fig. 1A,B). We imaged lobule VIA in P32-P34 mice presented with a randomized battery of sensorimotor stimuli ( $n=16$ mice, 82 FOVs). Mice were subjected to forced locomotion at a fast $(8 \mathrm{~cm} / \mathrm{s})$ or slow $(4 \mathrm{~cm} / \mathrm{s})$ speed on a motorized treadmill. Outside of the forced locomotion periods, animals were free to locomote at will, and this movement was recorded by a rotary encoder attached to the treadmill. Additional stimuli, selected to engage the diverse mossy fiber systems that project to the vermis, were presented during free-wheel periods and included air puffs to the face or trunk, flashing or sustained light, and a single tone beep or prolonged tone warble.

To identify granule neurons in lobule VIA displaying activated GCaMP6f signal, we used a custom cell segmentation algorithm (Fig. $1 B, C$ ). In addition, we validated the anatomical identity of the activated GCaMP6f segments in the high-density IGL as neuron somas upon expression of the fluorescent protein mCherry in a subset of granule neurons using an in vivo electroporation approach (Fig. 2A-D). Remarkably, this approach revealed that only $50 \%$ of active GCaMP6f ROIs that were mCherry-positive corresponded to mCherry-positive granule 
A
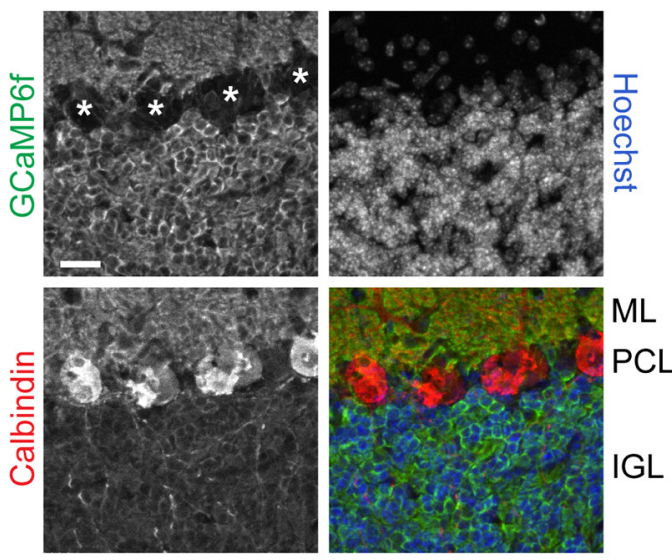

B

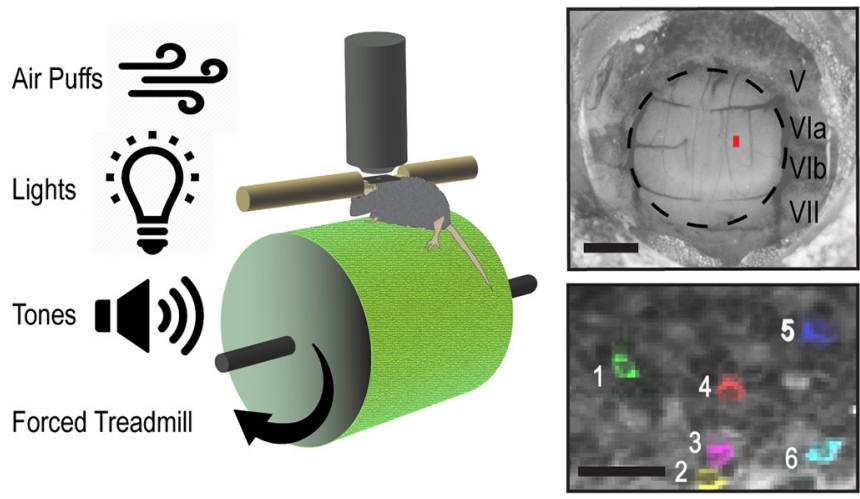

C

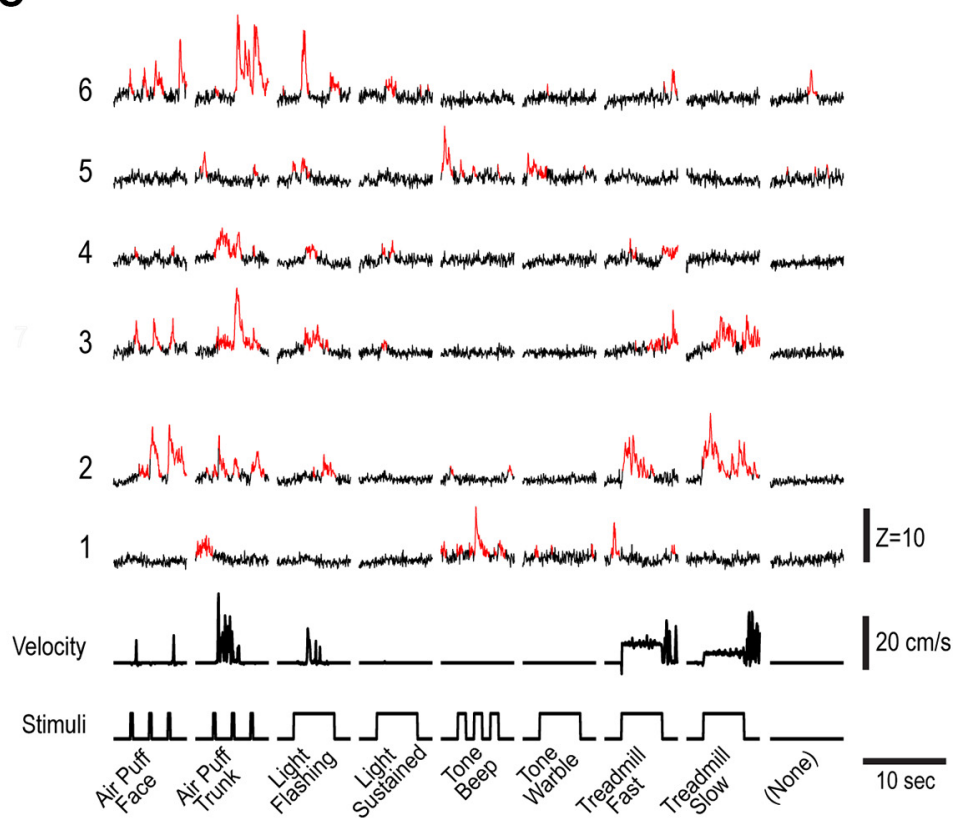

Figure 1. Imaging cerebellar granule neuron responses to in vivo sensorimotor stimuli. A, GCaMP6 ${ }^{d S L}$; GABA(A)R R 6-Cre mice were subjected to immunohistochemistry analyses using antibodies against GCaMP6f and calbindin as well as the DNA dye bisbenzimide (Hoechst 33258). GCaMP6f-positive rings had Hoechst-positive/GCaMP6f-cleared nuclei, and GCaMP6f was excluded from calbindin-positive Purkinje cells ( $^{*}$. Scale bar, $20 \mu \mathrm{m}$. PCL, Purkinje cell layer. $\boldsymbol{B}$, Schematic of in vivo imaging setup. Left, Head-fixed juvenile mice were subjected to a randomized sensorimotor stimulus battery of air puffs, lights, tones, and forced locomotion on a motorized treadmill. Right top, Cranial window was made over lobule VIA. Red rectangle represents the area of a typical FOV (mean 10,923 $\mu \mathrm{m}^{2}$ ). Two to seven fields were imaged per animal. Scale bar, $1 \mathrm{~mm}$. Right bottom, Mean GCaMP6 intensity of granule neurons imaged from GCaMP6 ${ }^{d S L}$; GABA(A)R $\alpha 6$-Cre mice during sensorimotor stimulation. Pseudocolored overlays represent ROls identified by an activity-based automated segmentation algorithm and manually verified to be nuclear- cell somas, whereas the remainder corresponded to cerebellar glomeruli, which include granule neuron dendritic claws (Altman, 1972; Billings et al., 2014) (Fig. $2 E, F)$. To further focus our analyses on somatic calcium activity, which reflects spiking of individual granule neurons (Giovannucci et al., 2017), rather than glomerular calcium activity, which reflects signals of several dozen granule neurons, we manually selected segments with unambiguous nuclear clearing in the GCaMP6f fluorescence channel (see Materials and Methods) (Wagner et al., 2017). Using these criteria, we selected 1375 granule neuron somas for analysis. Together, these steps established the feasibility to characterize large numbers of bona fide granule neuron somatic responses to sensorimotor stimuli in vivo.

\section{Identification of granule neurons that respond differentially to voluntary and forced locomotion}

We first evaluated how the activity of individual granule neurons in the developing mouse brain correlated with the gross locomotor movements detected by a rotary encoder. The rotary encoder detected treadmill movements both during epochs of fixed-speed treadmill rotation driven by a motor, and during epochs of freewheel rotation in which the motor clutch was disengaged and the treadmill speed was fully determined by the mouse's movements.

Movements occurred with varying likelihood and speed across all stimulus blocks (Fig. $3 A, B$ ). Some granule neurons were consistently activated in association with encoder-detected movements (Fig. 3C) (e.g., Cell 9, Pearson $r=0.39, p<$ 0.001 ), whereas others did not display consistent movement-associated activity (Fig. 3C) (e.g., Cell 7, Pearson $r=-0.13$, $p=0.31$ ). However, other neurons exhibited robust activation during a subset of movement epochs and remained completely silent during other epochs (Fig. 3C) (e.g., Cells 8 and 10). Remarkably, some of these neurons appeared to respond selectively either to movements during the motorized-treadmill epochs

cleared granule neuron somas. Scale bar, $25 \mu \mathrm{m}$. C, Mice were presented with eight stimulus blocks in a pseudorandomized order while velocity and granule neuron calcium activity were recorded. Z-scored calcium activity traces of six nuclearcleared somas (from $\boldsymbol{B}$ ) are depicted for one episode of each stimulus block. Red represents statistically significant transients. Bottom, Recorded velocity trace and stimulus time courses. 
A

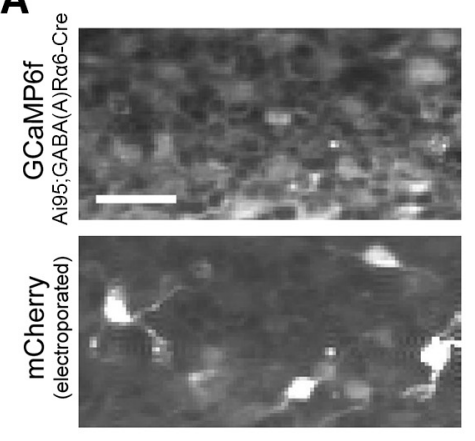

B

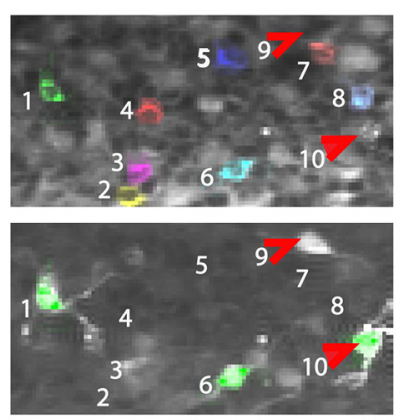

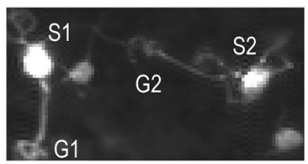

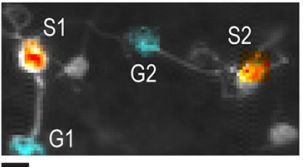

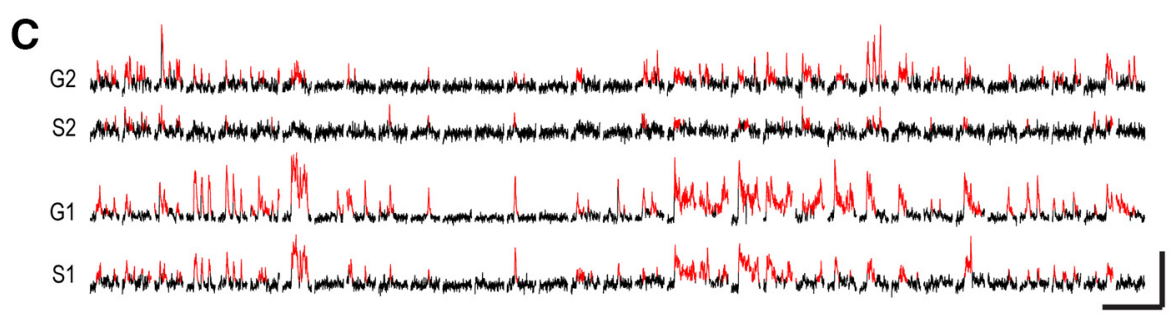

D

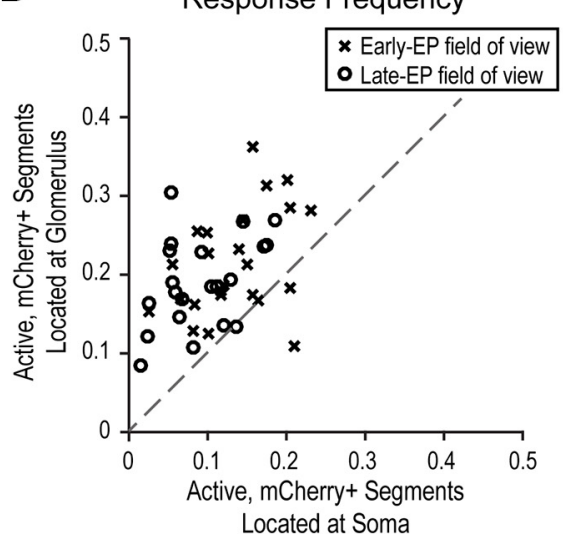

$\mathbf{E}$

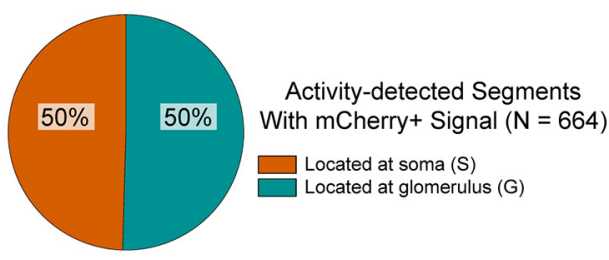

$\mathbf{F}$

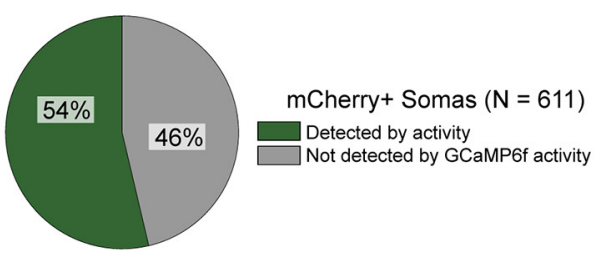

Figure 2. Calcium imaging of responses in bona fide granule neuron somas. $A$, Sample results of granule neuron detection by activity-based cell segmentation algorithm and manual assessment of nuclear clearing. Mean GCaMP6f intensity (top) and mCherry intensity (bottom) from an imaged region of the IGL without (left) and with (right) pseudocolored overlay of granule neurons detected by activity-based segmentation. Image is an expanded FOV from Figure 1B. Top right, Cells 1-8 are examples of cells included in sensorimotor coding analyses (Figs. 3-6) due to detection by activity-based segmentation algorithm and manual verification as nuclear-cleared in the GCaMP6f channel. Bottom right, Examples of three cells $(1,6,10)$ included in birthdate analyses (Fig. 8) due to detection by activity-based segmentation algorithm and coincidence with $\mathrm{mCherry-positive} \mathrm{birthdate-labeled} \mathrm{granule} \mathrm{neuron} \mathrm{somas.} \mathrm{Open} \mathrm{arrowhead} \mathrm{indicates} \mathrm{granule} \mathrm{neurons} \mathrm{that} \mathrm{were}$ identified by their mCherry signal but lacked detectable GCaMP6f activity under the imaged conditions and thus were not analyzed. Filled arrowhead indicates granule neuron somas that were detected by GCaMP6f activity and verified by mCherry signal to correspond with a granule neuron soma but were not identified as nuclear-cleared; these somas were only included in birthdate analyses (Fig. 8). Scale bar, $25 \mu \mathrm{m}$. B, Examples of ROls identified by the activity-based cell segmentation algorithm that coincided with granule neurons sparsely labeled by electroporation of a pCAG-mCherry plasmid in GCaMPGFISL; GABA(A)R R6-Cre mice. Some active ROls (orange) coincided with mCherry-positive granule neuron somas (S), but others (cyan) were located at mCherrypositive dendritic claws in the glomerulus region (G). Scale bar, $10 \mu \mathrm{m}$. C, Traces of GCaMP6f activity from ROls located over somas and glomeruli of the mCherry-labeled granule neurons in $\boldsymbol{B}$. Red traces represent epochs of statistically significant calcium transients. Calibration: $20 \mathrm{~s}, Z=10$. D, Response frequency was calculated for all ROls as the proportion of recorded time during which statistically significant transients occurred. Mean response frequencies for ROIs located at mCherry-positive somas and glomeruli are plotted for each FOV containing at least four examples of each ROI type. Data include FOVs from mice electroporated at early (Early-EP) and late (Late-EP) time points. ROls located at mCherry-positive glomeruli had higher response frequencies than ROIs at $\mathrm{mCherry}$-positive somas. $E$, The ROIs detected by activity-based segmentation and found to overlap with an $\mathrm{mCherry}$-positive granule neuron were compared with the $\mathrm{mCherry}$ signal to determine whether each ROI corresponded to a granule neuron soma or a glomerulus $(n=664)$. $\boldsymbol{F}$, The mCherry-positive granule neurons recorded during sensorimotor stimulus presentations were compared with the automated segmentation algorithm to determine how many of the sparsely labeled somas were detected by activity-based segmentation ( $n=611$ neurons from $82 \mathrm{FOV}$ in 16 mice).

(e.g., Cell 8) or else to movements during the free-wheel epochs (e.g., Cell 10).

To quantify the movement response selectivity of each cell, we calculated a free-wheel responsivity index and a motorized-wheel responsivity index, each corresponding to the fraction of time that calcium transients occurred within the subset of frames exhibiting the respective movement context. Across 1196 granule neurons, the free-wheel and motorized-wheel indices were not deterministic of each other (Fig. 3D) (Pearson $r=0.55, p<0.01$ ).
Notably, granule neuron responses filled the entire space of possible combinations of the parameters of free-wheel and motorized-wheel responsivities (Fig. 3D). Furthermore, neurons recorded concurrently in the same FOV exhibited diverse movement response selectivities (Fig. 3E), suggesting that the differential responses to locomotion in free-wheel versus motorized-wheel contexts were not primarily due to behavioral variance between trials. Together, these results suggest that granule neurons exhibit diverse response selectivities to locomotion 
A

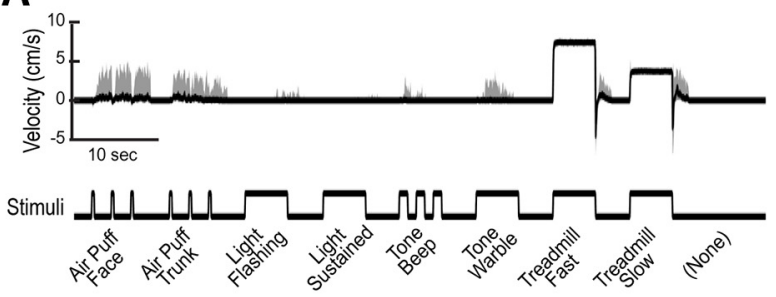

B

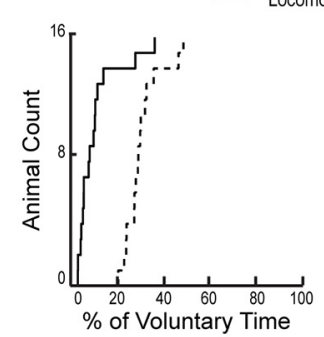

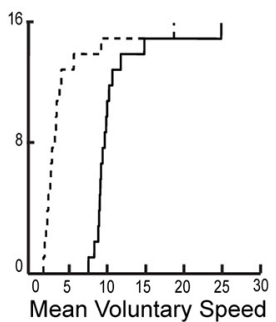

c

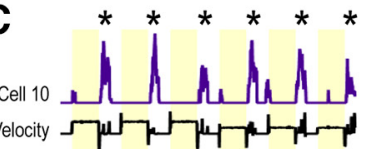

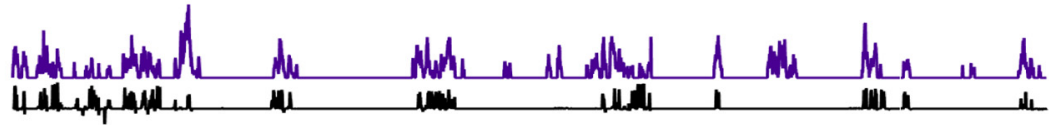
Cell 9 , than Velocity 5 (1)

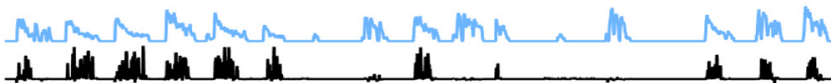

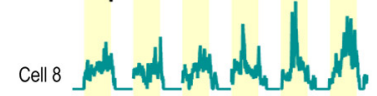

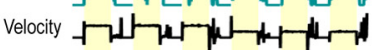

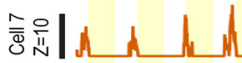

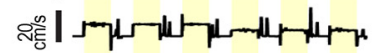
$10 \mathrm{~s}$

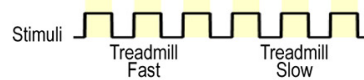

Motorized Wheel

D

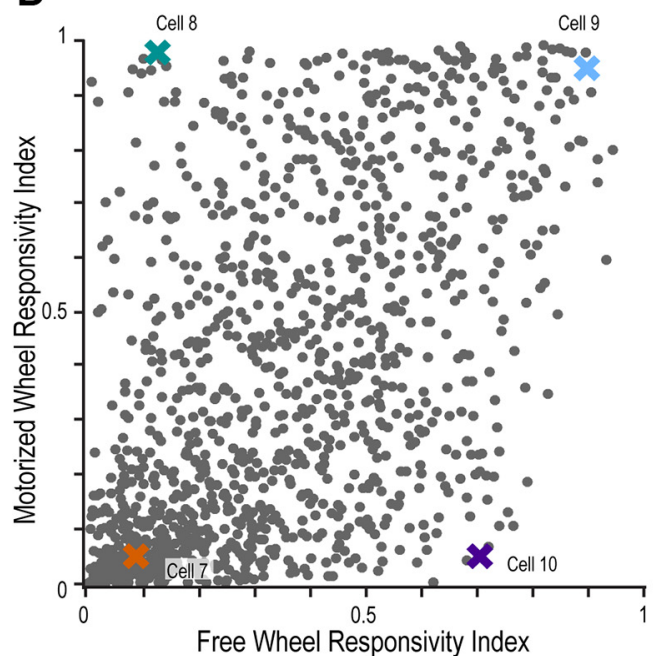

E

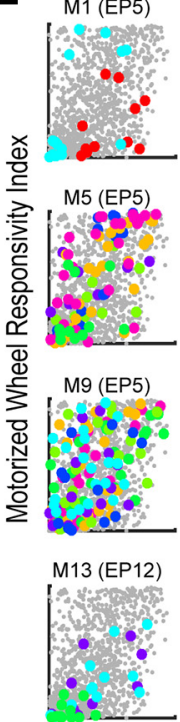

M2 (EP5)
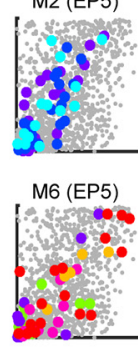

M10 (EP12)

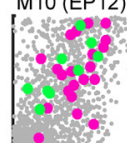

M14 (EP12)

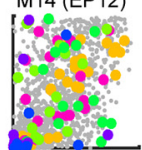

Free Wheel Responsivity Index

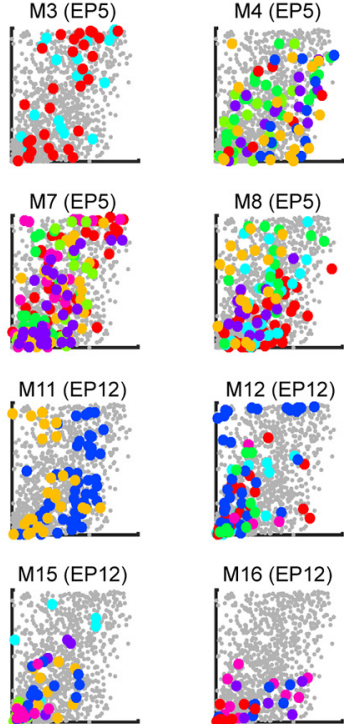

Figure 3. Granule neurons respond differentially to voluntary and forced locomotion. $A$, Variability in treadmill velocity recorded during presentations of sensorimotor stimuli. Median (bold line) and interquartile range (gray shading) of velocities in 82 FOVs from 16 mice. Three trials of each stimulus were recorded per FOV. B, Cumulative distribution plots of the percentage of time imaged under voluntary wheel control during which each mouse exhibited voluntary movements (non-0 velocity) or locomotion (velocity $>3 \mathrm{~cm} / \mathrm{s}$ ) (left) and the mean speed (cm/s) during those times (right) ( $N=16$ mice). C, Calcium activity and treadmill velocity data during stimulus blocks with motorized-wheel trials (left) and free-wheel epochs (right) for four example cells that exhibit distinct response selectivities to voluntary versus forced movements. Yellow patches represent epochs of motorized wheel-ON. Trials were presented in a pseudorandomized fashion but are ordered for display. *Neural responses during motorized-wheel stimulus blocks that occurred during interstimulus epochs when the motor was $0 \mathrm{FF}$ and the wheel was under voluntary control. $\boldsymbol{D}$, Relationship between free-wheel responsivity index and motorized-wheel responsivity index for granule neurons in lobule VIA ( $n=1196$ activity-detected, nuclear-cleared somas from 16 mice). Example cells from $\boldsymbol{C}$ are highlighted. $\boldsymbol{E}$, Free-wheel and motorized-wheel responsivity indices, plotted as in $\boldsymbol{D}$, with the neurons recorded from each mouse denoted in color and neurons from all other mice denoted in gray. Within each mouse (M), neurons from different FOVs are shown in distinct colors. Mice were electroporated at postnatal day 5 (EP5) or 12 (EP12) and imaged between P32 and P34. 
A

\section{Known Variables}

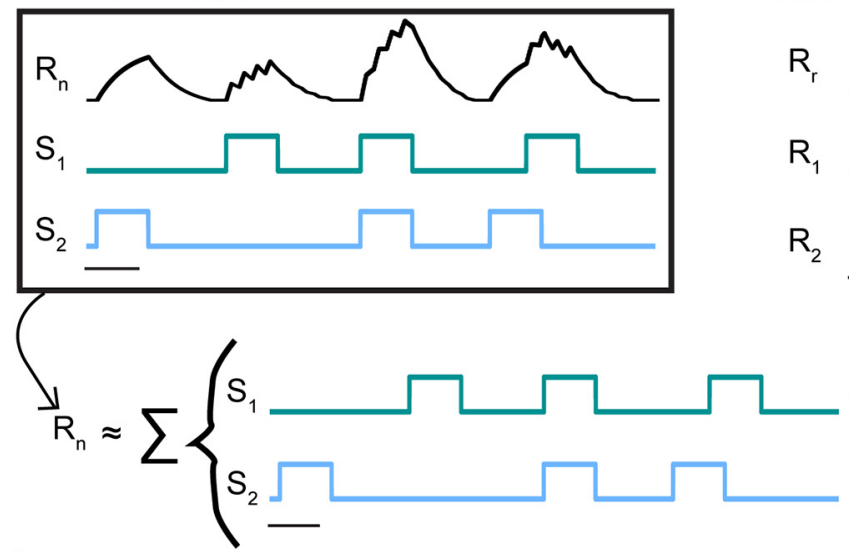

B

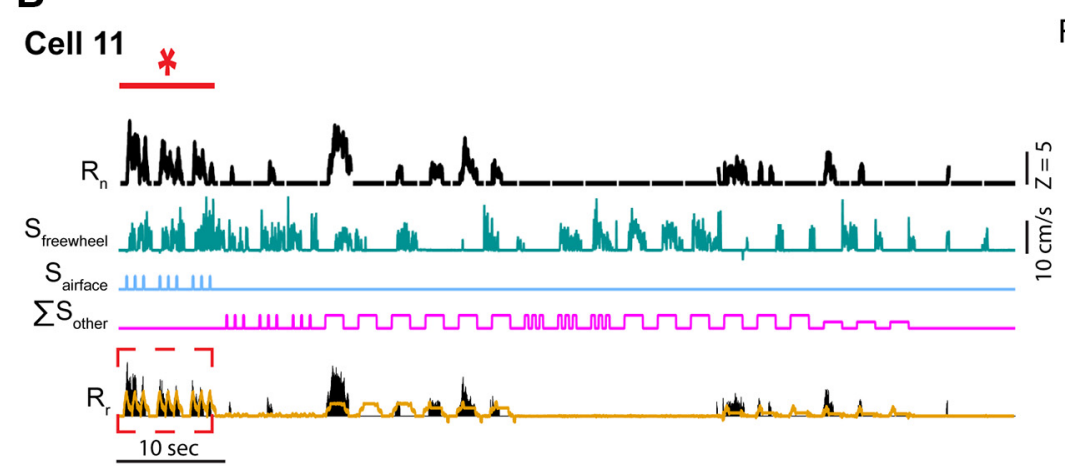

Reconstructed Response: $R_{n} \approx R_{r}=R_{1}+R_{2}$

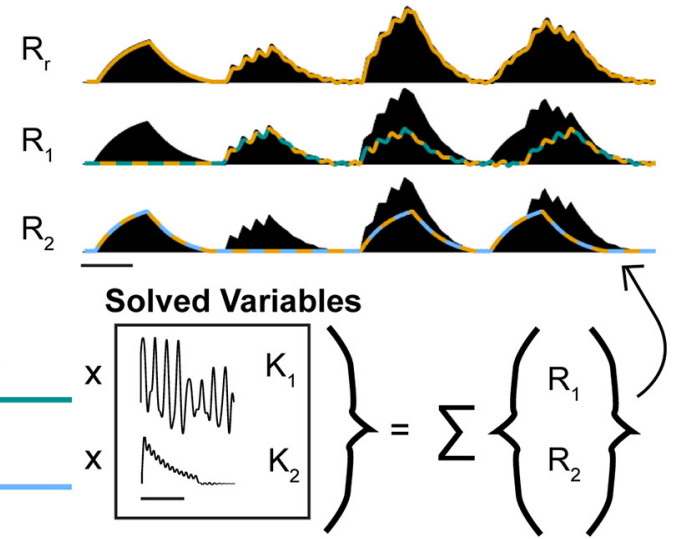

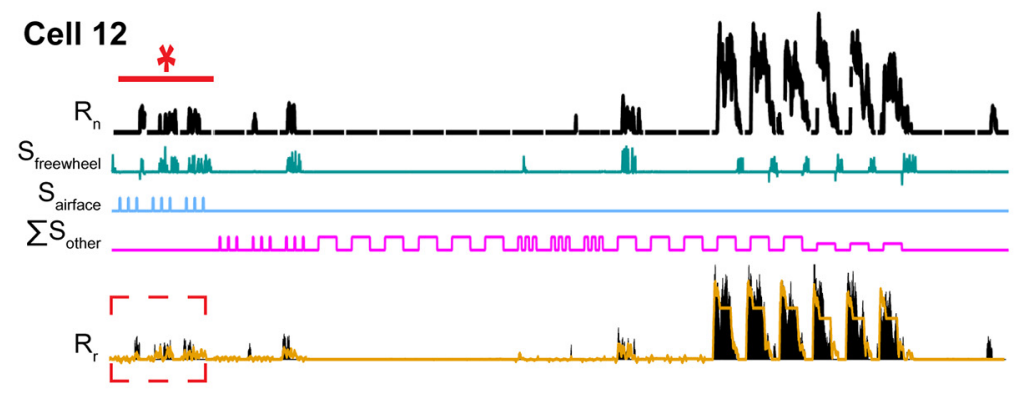

$\mathrm{R}_{\mathrm{r}}=\mathrm{R}_{\text {freewheel }}+\mathrm{R}_{\text {airface }}+\sum \mathrm{R}_{\text {other }}$

$R_{r} M$ $R_{\text {treemeel }}$ Wh the Wh

$R_{\text {atros }}$ M M M M M

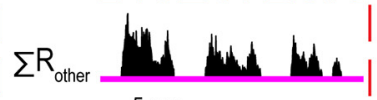

$\left\llcorner-\frac{5 \mathrm{sec}}{-}-\ldots\right.$

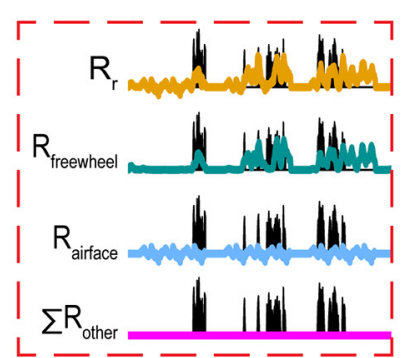

C

Wheel Movement (Free \& Motorized)
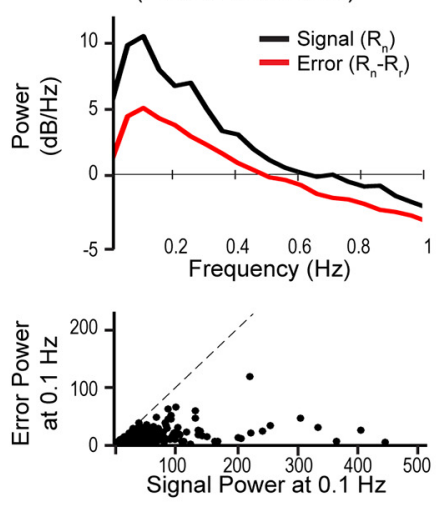

D

All Stimuli
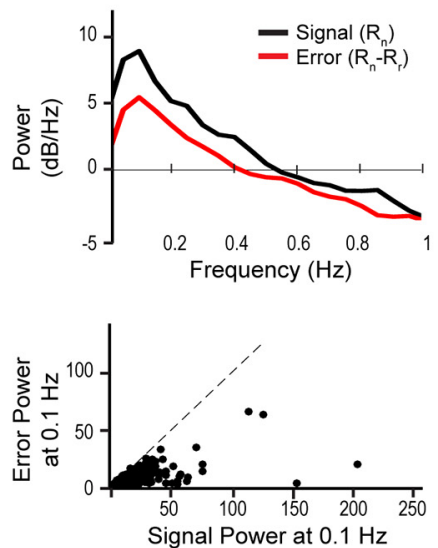

E
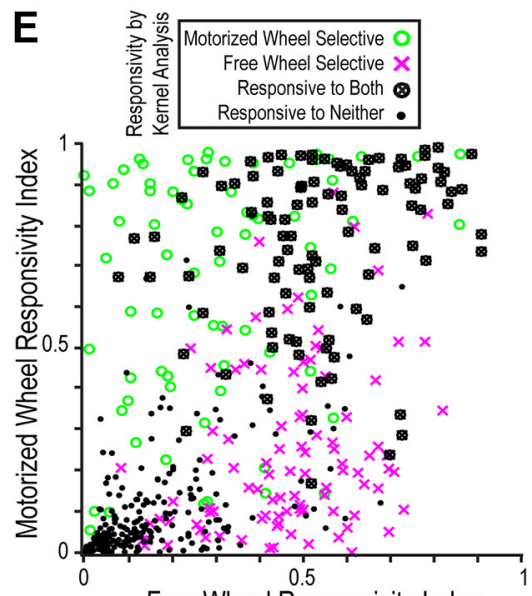

Free Wheel Responsivity Index

Figure 4. A multikernel linear model disambiguates neural responses to sensorimotor stimuli and co-occurring movements. $A$, Schematic of the multikernel linear model analysis. A recorded neural response $\left(R_{n}\right)$ and known stimuli with partially overlapping time courses $\left(S_{1}, S_{2}\right)$ are used to determine the portion of neural activity explained by each stimulus. Stimulus-specific kernels $\left(K_{1}\right.$, $\mathrm{K}_{2}$ ) are determined analytically by minimizing the sum of least square errors (see Materials and Methods) such that, once convolved with their respective stimuli, the stimulus-specific responses ( $R_{1}$, $R_{2}$ ) sum to a reconstructed response $\left(R_{r}\right.$ yellow line) that best fits the recorded response $R_{n}$ (shaded black area). Calibration: $1 \mathrm{~s}$. $\boldsymbol{B}$, Results of the multikernel linear model analysis are shown for two granule neurons with recorded neural responses $\left(R_{n}\right)$ during the facial air puff stimulus blocks (red asterisk) that could be attributed to the air puff stimulus $\left(S_{\text {airface }}\right)$ or the c0-occurring voluntary movements $\left(S_{\text {freewheel }}\right)$ during those blocks. The seven other sensorimotor stimuli do not occur during this epoch and have been summed $\left(\Sigma S_{\text {other }}\right)$ for condensed (Figure legend continues.) 
on a freely rotatable versus motor-driven treadmill. Importantly, however, the different amount of temporal variation present in free versus forced locomotion as well as the intermittent coincidence of free-wheel locomotion with presentation of other external stimuli remained as possible explanations for the context-selective responses. These limitations motivated us to develop a more sophisticated method capable of disentangling and describing the stimulusresponse properties of cerebellar granule neurons.

\section{A multikernel linear model disambiguates neural responses to sensorimotor stimuli and co-occurring movements} To determine whether granule neurons indeed respond differentially to free and forced locomotion, we developed an encoding model that captures a range of kinetic responses. The model also presented the opportunity to address a major hurdle that plagues imaging studies of cerebellar responses in mice in vivo. Because presentations of external stimuli evoke variable amounts of locomotor movement (e.g., Fig. 3A), granule neuron responses to stimuli are often contaminated with responses to co-occurring movement. Observed correlations between stimuli and neural activity may thereby reflect neural encoding of the stimulus, the associated movements, or both. To resolve such ambiguities, we developed a multikernel linear model that takes into account all known stimuli and describes the recorded neural response as a combination of responses to the individual stimuli (Fig. 4A). This model is based on the principle that a recorded stimulus-evoked calcium signal, which reflects the convolution of the neuron's firing rate with the calcium indicator's time course, can be approximated as the convolution of the known stimulus time course with an analytically determined cell/stimulus-specific kernel (Simoncelli et al., 2004; Dayan and Abbott, 2005). Thus, using the known stimulus time courses and the recorded composite neural response of each granule neuron, the optimal set of stimulus-specific kernels was determined for each neuron such that the net reconstructed response was best fit to the recorded response (Fig. 4A).

The example cells in Figure $4 B$ illustrate the utility of the multikernel linear model for disambiguating granule neuron responses to co-occurring stimuli. Both example granule neurons exhibit neural activity during the facial air puff peristimulus period, but mouse body movements during this same period cast doubt as to whether the recorded neural activity reflects responsivity of granule neurons to the air puff itself or to the cooccurring movements. Using information across all trials and stimuli, the model deduced that neural activity in Cell 11 occurred in response to the air puff itself, whereas neural activity in Cell 12 occurred in response to the voluntary movements. These

\footnotetext{
(Figure legend continued.) presentation. Right, Reconstructed stimulus-specific responses reveal that the recorded activity (shaded black area) during air puff blocks is best explained as a response to the air puff stimulus in Cell 11 and as a response to the associated free-wheel movements in Cell 12. C, Quantification of the accuracy of the model in explaining the recorded neural response. Top, Plot represents the average power spectrum of the true signal $\left(R_{n}\right)$ and predictive error (error $=R_{n}-R_{r}$ ) during treadmill stimulus blocks ( $n=6$ trials per neuron, 1271 neurons). The model performs best at low frequencies, as also illustrated schematically in A. Bottom, Plot of the signal power and error power at $0.1 \mathrm{~Hz}$ for the individual cells contributing to average in the top panel. The predictive error remains low for the majority of neurons, regardless of their signal power. $\boldsymbol{D}$, Power analysis as in $\mathbf{C}$ for data in all stimulus blocks. The model performs best at low frequencies. $\boldsymbol{E}$, Relationship between free-wheel responsivity index and motorized-wheel responsivity index for granule neurons, as in Figure 3D, with the color of each datapoint denoting the responsivity of each neuron determined by the multikernel linear model analysis.
}

interpretations are consistent with visual inspections of the responsivity of each granule neuron to voluntary movements during nonair puff periods (Fig. 4B).

To assess the accuracy of the model, we first examined how well it reconstructed the recorded neural activity during trials that included wheel movements. Among all granule neurons analyzed, the model reconstructed the low-frequency components of calcium activity with high fidelity $(71 \%$ accuracy at $0.1 \mathrm{~Hz})$ (Fig. 4C), demonstrating its ability to accurately identify epochs when each cell is activated. As expected, the model was less capable of reconstructing higher frequency responses (39\% accuracy at $0.4 \mathrm{~Hz}$ ) (Fig. 4C), which may arise from the variable, intermittent bursting patterns of granule neurons (Duguid et al., 2015; Powell et al., 2015; Witter and De Zeeuw, 2015) and from behavioral variability between movement epochs (Lisberger and Medina, 2015). The model was predictive during presentations of other sensorimotor stimuli as well (Fig. 4D), thus confirming its ability to capture dominant components of granule neuron activity across a variety of conditions.

Having verified its predictive power, we next used the results of the multikernel linear model analysis to determine how granule neurons respond to free versus forced locomotion. In addition to evaluating movements time-locked with calcium activity, as in the prior calculation of free-wheel and motorized-wheel responsivity indices, the kernel analysis facilitated assessment of more complex kinetic relationships between stimulus history and neural activity and disambiguated responses to stimuli that partially overlap in time (Fig. $4 B$ ). Using this analysis, we found that $44 \%$ of active granule neurons were responsive to free locomotion and $51 \%-56 \%$ were responsive to forced locomotion. Strikingly, among responsive cells, some individual neurons responded selectively to either free or forced locomotion (Fig. 4E), and these classifications corroborate the results of our responsivity analysis based on free-wheel and motorized-wheel responsivity indices. Collectively, based on the distinct approaches of responsivity index and kernel analysis, we conclude that granule neurons differentially respond to locomotion in voluntary versus forced contexts.

\section{Granule neurons in the vermal cerebellum encode temporally diverse features of sensorimotor stimuli}

By disentangling the influence of co-occurring stimuli on neural activity, the multikernel model enabled more accurate assessment of whether and how granule neurons in lobule VIA responded to each sensorimotor stimulus. Each of the nine stimuli assessed in vivo was associated with activity in a subset of granule neurons (Fig. 5A). Forced locomotion and facial air puffs each triggered responses in approximately half of the active subset of granule neurons (fast treadmill: $51 \%$, slow treadmill: $56 \%$, facial air puff: $50 \%$ ), whereas free locomotion (44\%), visual (light flashing: 32\%, light sustained: $31 \%$ ), and auditory (tone beep: $19 \%$, tone warble: $28 \%$ ) cues stimulated smaller proportions of the active neurons (Fig. 5A). Because labeling neurons with an activity-independent marker by in vivo electroporation suggested that only $54 \%$ of vermal granule neurons were detectable by GCaMP6f activity-based segmentation (Fig. $2 F$ ), these data indicate that each sensorimotor stimulus activated between $10 \%$ $(0.19 \times 0.54)$ and $30 \%(0.56 \times 0.54)$ of all granule neurons in lobule VIA.

Although subsets of granule neurons were identified as responsive to each stimulus, the consistency with which responses were evoked across multiple trials varied (Fig. $5 A$ ). Neurons responsive to a facial air puff or motorized treadmill stimulus were 
most likely to have high-fidelity responses, defined as responses to at least two of three trials. In contrast, the majority of neurons identified as responsive to the visual or auditory stimuli had statistically significant responses to only one of the three trials.

Among the subset of neurons with high-fidelity responses to a given stimulus, the time course of stimulus-evoked calcium activity was often similar between trials, but different between neurons. For example, each presentation of a facial air puff evoked a sharp onset and rapid decay of calcium activity in Cell 13, whereas in Cell 14 each facial air puff was associated with a calcium transient that still had a rapid onset but then remained elevated for 1-2 s after air puff termination (Fig. $5 B)$. Importantly, these temporally diverse responses were recorded simultaneously from granule neurons in a single FOV, indicating that the different response time courses were not primarily due to trial-to-trial behavioral differences.

To assess the variety of response time courses for all air puff-responsive granule neurons, we convolved each neuron's computed air puff response kernel with the air puff stimulus time course. The resulting "characteristic response" for each neuron represents the neuron's typical response to a single air puff presentation. A heatmap of characteristic responses for all responsive neurons thus illustrates the proportion of neurons exhibiting different calcium time courses (Fig. 5C). Forced locomotion also evoked high-fidelity responses from many granule neurons, and the time courses of these responses also showed relative consistency between trials but diversity between neurons. For example, the calcium activity of some granule neurons peaked shortly after treadmill initiation and subsequently decayed (e.g., Cell 15), whereas other neurons had calcium activity that remained elevated throughout the duration of the treadmill-on epoch (e.g., Cell 16), or activity that increased following termination of the treadmill and return to the free-wheel state (e.g., Cell 17) (Fig. 5D,E). The diversity of characteristic responses suggests that the subset $(10 \%-30 \%)$ of vermal granule neurons responsive to each stimulus encodes multiple different stimulusassociated features or encodes a single feature with a variety of temporal kinetics.
A

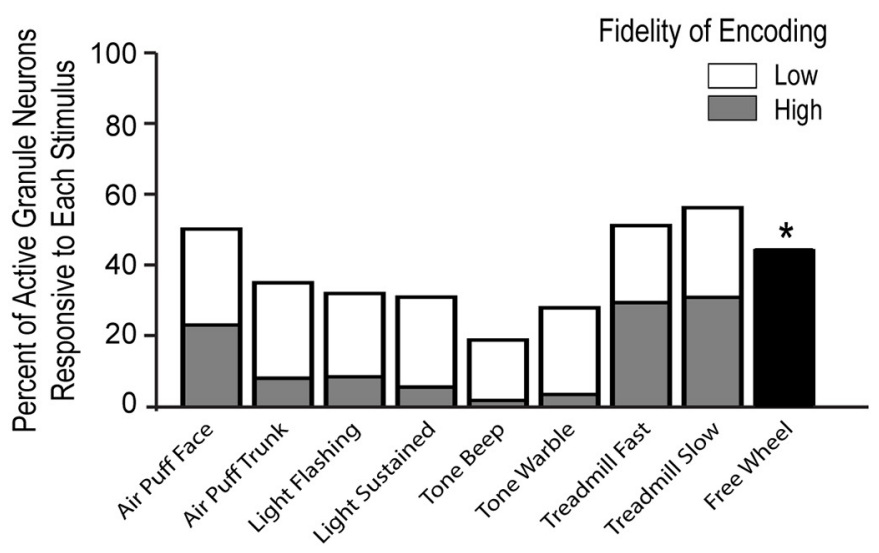

B

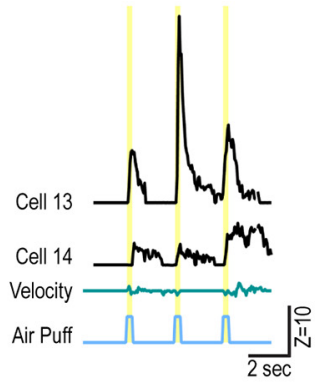

D

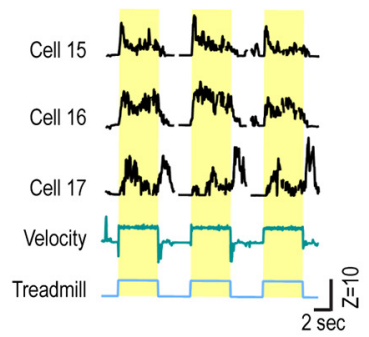

C

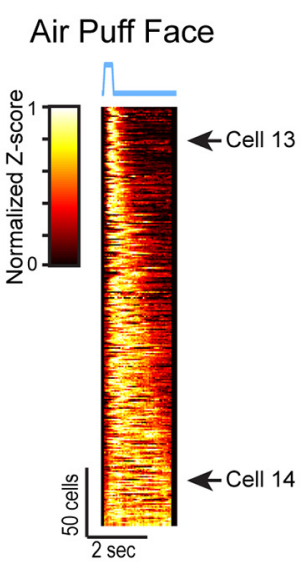

$\mathbf{E}$

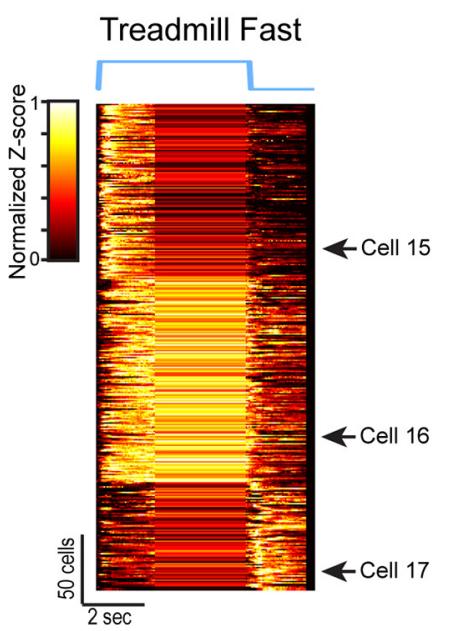

Figure 5. Granule neurons encode temporally diverse features of sensorimotor stimuli. $A$, Responsivity to sensorimotor stimuli was determined for a population of 1375 active granule neurons using a multikernel linear model analysis. Histogram represents the proportion of active granule neurons that was statistically significantly responsive to each stimulus ( $n=1147-1375$ neurons per condition; only neurons with three trials of the stimulus were included in the analysis). Shading represents fidelity of neural response, assessed over three stimulus trials. *Free-wheel responsivity was assessed from data in multiple stimulus blocks (see Materials and Methods) and therefore did not have discrete trial structure needed for calculation of response fidelity. $\boldsymbol{B}$, Raw neural responses of two granule neurons recorded simultaneously that exhibit distinct time courses of activation following presentation of facial air puff stimulus. C, Characteristic neural responses to the facial air puff stimulus were calculated by convolving each cell/stimulus-specific kernel with the time course of a single air puff trial. A heatmap of the normalized characteristic responses for 293 neurons activated by at least two of the three stimulus blocks is shown, ordered by the FWHM of their responses. D, Raw neural responses, as in $\boldsymbol{B}$, for three simultaneously recorded granule neurons that respond differentially to the fast treadmill stimulus. $\boldsymbol{E}$, Characteristic neural response to the fast treadmill stimulus, as in $\boldsymbol{C}$. For display purposes, neurons are ordered after $k$-means clustering $(k=3)$. The vertical lines apparent 2 s after stimulus initiation and at the moment of stimulus termination arise because each individual cell's predicted response has a constant value on this interval, an expected consequence given the 2 s kernel length (see Materials and Methods).

Individual granule neurons in the vermal cerebellum respond to diverse combinations of sensorimotor stimuli

Response characteristics in the IGL are constrained not only by the type of information carried by mossy fibers but also by the arrangement of those afferent fibers onto individual granule neurons (Ekerot and Jörntell, 2008; Arenz et al., 2009; Huang et al., 2013; Gilmer and Person, 2017). Because some granule neurons receive three to five functionally similar unimodal inputs (Jörn- 
A
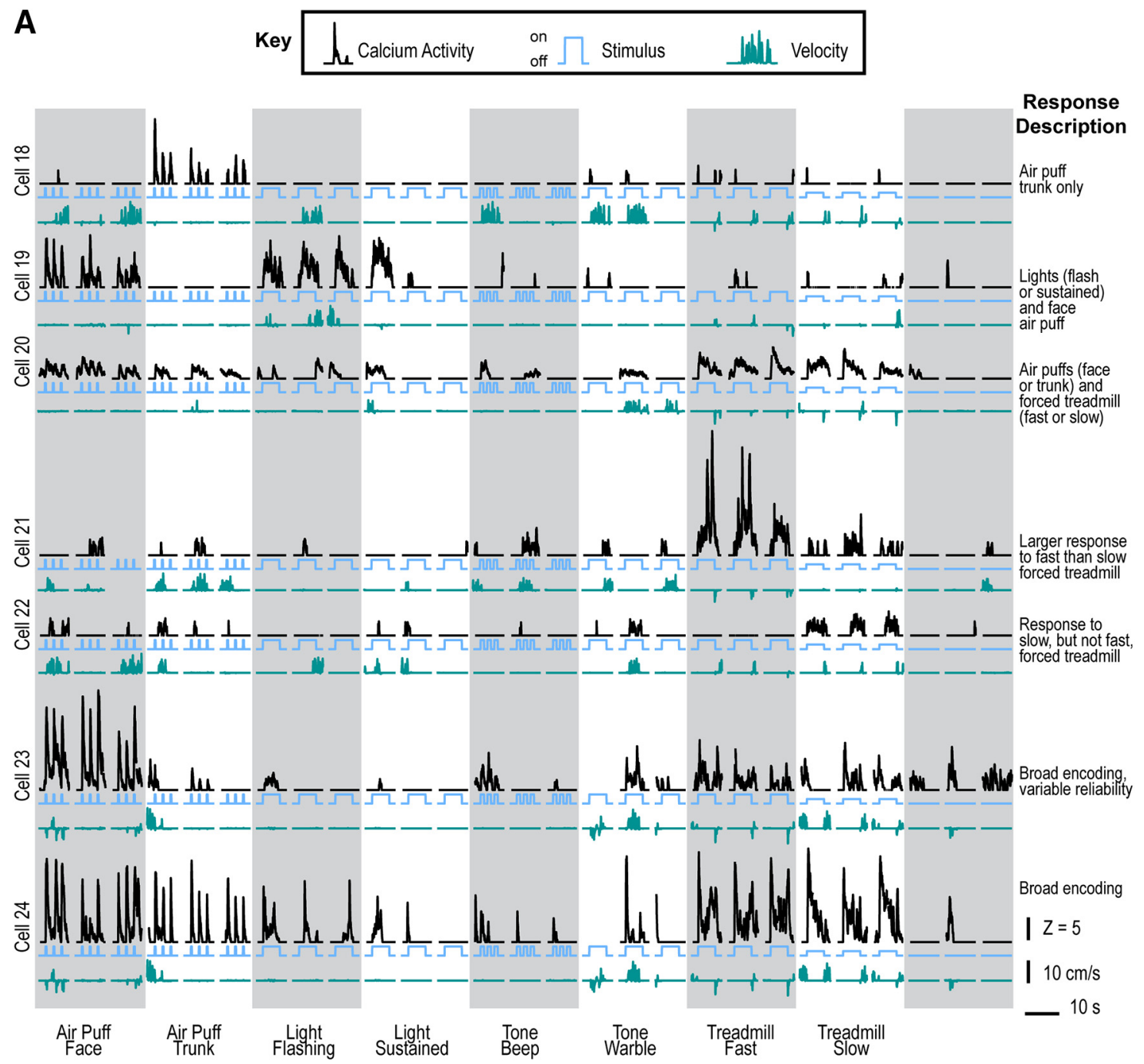

B

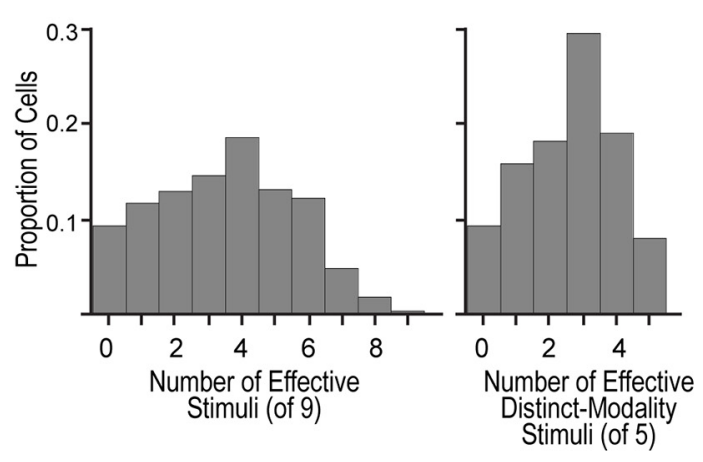

C

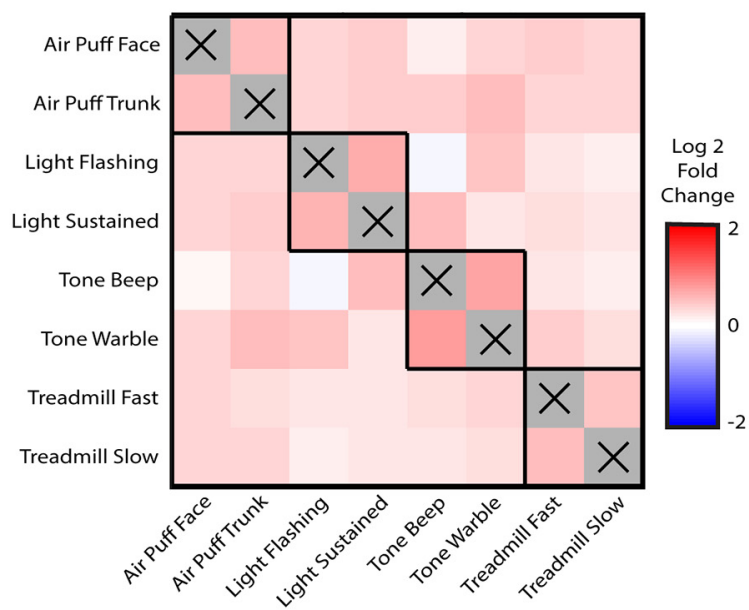

Figure 6. Granule neurons encode diverse combinations of sensorimotor stimuli. $A$, Representative granule neuron responses to distinct sensorimotor stimuli were chosen to highlight the variety of response combinations observed. To ease direct visual interpretation, sample neurons were preferentially selected from recordings without extensive voluntary movements. $\boldsymbol{B}$, Responsivity to nine sensorimotor stimuli was calculated for granule neurons using a multikernel linear model approach. Left, Histogram of the number of stimuli to which each granule neuron responded ( $n=834$ neurons; only neurons with three trials of all stimuli were included in the analysis). Right, Responses to the pairs of sensorimotor stimuli targeting similar sensory modalities (i.e., light flashing or sustained, tone beep or warble, air puff to face or trunk, and forced treadmill fast or slow) were combined. Histogram represents the number of distinct-modality stimuli to which each granule neuron responded. C, The proportion of granule neurons responsive to one stimulus that also responded to a second stimulus was calculated. Heatmap represents overabundance or underabundance of neurons with each dual responsivity relative to the expected abundance of responses to the second stimulus in the total population (log2-transformed values). Black boxes represent dual responses to the pairs of stimuli activating similar sensory modalities. Gray shading with black X's represents the location where each stimulus intersects with itself; the probability of responding to a "pair" of stimuli is not meaningful in these redundant cases. 
tell and Ekerot, 2006; Bengtsson and Jörntell, 2009) and others receive dissimilar multimodal inputs (Huang et al., 2013; Chabrol et al., 2015; Ishikawa et al., 2015), we evaluated whether and how granule neurons in lobule VIA respond to multiple sensorimotor cues. Although a subset of neurons responded to only one of the sensorimotor stimuli (Fig. 6A) (e.g., Cell 18, trunk air puff), the majority of granule neurons responded to multiple stimuli (Fig. 6A). These included neurons with responses to pairs of stimuli that engage the same sensory modality (e.g., Cell 19, light flashing or sustained; Cell 20 and 21 treadmill fast or slow; Cell 24 air puff to face or trunk), as well as neurons responsive to stimuli that engage distinct sensory modalities (e.g., Cells 19, 20, 23, and 24). Interestingly, granule neurons differed in their relative responses to pairs of stimuli engaging the same modality. For example, in response to the slow versus fast motorized treadmill, neurons exhibited similar (e.g., Cell 20, 24), disparate (e.g., Cell 21), or selective (e.g., Cell 22) responses. Likewise, some cells responded selectively to an air puff of the face (Cell 19) versus trunk (Cell 18), whereas others responded to both air puff stimuli (Cells 23 and 24), sometimes with differing reliabilities (Cell 23). Together, these examples highlight the variety of features encoded at the level of individual granule neurons in the vermal cerebellum and underscore the increased neuronal diversity that is apparent in the granule neuron population upon consideration of responses in multiple functional domains.

Across the population of active granule neurons, 79\% exhibited responses to two or more of the nine cues experienced by the mice (Fig. 6B). Notably, even after discounting neurons that responded only to a pair of same-modality cues (e.g., light flashing and light sustained), the majority of neurons (75\%) were activated in response to multiple distinct-modality stimuli (Fig. 6B).

Insofar as distinct functional responses reflect input from distinct mossy fibers, the functional code of the granule neuron population may provide insight into patterns of mossy fiber connectivity. We analyzed whether particular combinations of functional responses were overrepresented or underrepresented compared with chance, which might suggest nonrandom, biased mossy fiber connectivity. For every combination of two stimuli, we calculated the observed probability that a granule neuron responsive to Stimulus A also responded to Stimulus B. We then compared this dual-responsivity rate to the population-wide probability that any neuron responded to Stimulus B. Overall, granule neurons responsive to one external stimulus were more likely than the total population to respond to a second external stimulus (Fig. 6C). The enrichment was most pronounced among the four pairs of stimuli that targeted a common sensory modality (mean enrichment 52\%; Fig. $6 \mathrm{C}$, bold boxes) but was also present for pairs of sensorimotor stimuli targeting distinct modalities (mean enrichment 19\% among 24 dissimilar pairs of stimuli; Fig. 6C). Collectively, these results demonstrate that individual vermal granule neurons are frequently activated in association with multiple sensorimotor stimuli, suggesting nonrandom mossy fiber patterning or convergence of information upstream of the cerebellum.

\section{Early- and late-born granule neurons convey diverse sensorimotor information to spatially distinct parallel fiber domains in the cerebellum in vivo}

Having elucidated the characteristics of vermal cerebellar granule neuron responses to diverse sensorimotor stimuli in juvenile mice, we next asked how these response patterns were established and organized during brain development. Granule neuron responses to multiple sensorimotor stimuli may reflect inputs from multiple mossy fibers that emanate from distinct precerebellar nuclei. During development, mossy fibers of different precerebellar nuclei are born at different time points and the subsequent arrival of these fibers to the cerebellar cortex is also temporally staggered (Altman and Bayer, 1987, 1997; Ashwell and Zhang, 1992). Furthermore, within the cerebellar cortex, mossy fibers continue to grow and retract (Kalinovsky et al., 2011), and they respond to cues from developing granule neurons (Zhang and Mason, 1998). To test whether the timing of a granule neuron's birth during this dynamic early postnatal period affects its ultimate afferent connectivity, we therefore assessed the effect of the birth order of granule neurons on their responsivity to sensorimotor stimuli. Taking advantage of the prolonged generation of cerebellar granule neurons throughout the first two postnatal weeks in mice, we used in vivo electroporation at different postnatal time points to selectively label birth dated subpopulations of granule neurons. Analyses at P22 of granule neurons from mice that had been electroporated as early as P5 or as late as P15 revealed successful labeling of large cohorts of granule neurons in the targeted lobules IV/V, VI, and VII of the developing mouse brain (Fig. 7A).

The somas of labeled early- and late-born granule neurons were distributed throughout the IGL independently of birth order (Zong et al., 2005; Espinosa and Luo, 2008) (Fig. 7B). Remarkably, however, granule neuron parallel fibers of early- and late-born granule neurons were each stacked together at a specific depth within the molecular layer (ML) (Zong et al., 2005; Espinosa and Luo, 2008) (Fig. 7C). Quantification across cohorts of electroporated animals revealed that parallel fibers of early-born granule neurons (electroporated at P5) resided in the deep ML, near Purkinje cell somas, whereas neurons labeled at later time points (up to P15) occupied progressively more superficial positions within the ML (Fig. 7D). Notably, the timing of this sequential parallel fiber stacking differed between lobules in a stereotyped manner (Fig. 7D) (two-way ANOVA with main effect of lobule: $F_{(2,18)}=27.88, p<0.001$; main effect of electroporation age: $\left.F_{(3,9)}=22.63, p<0.001\right)$. The structure of the cerebellar cortex and thickness of the ML at P22 had little or no difference between mice that had been electroporated at early versus late time points (Fig. 7E) (one-way ANOVA: $F_{(3,12)}=0.87$, $p=0.49$ ). These results reveal that granule neuron parallel fibers are positioned in specific sublayers of the ML depending on their birth date. Foreshadowing these findings, Cajal's illustrations of the mammalian cerebellar cortex depict a relationship between granule neuron morphologic maturity and parallel fiber position in the ML (Ramon y Cajal, 1911). Clonally related genetically labeled granule neurons in the mouse cerebellum also display spatially clustered parallel fibers that depend on birth order (Espinosa and Luo, 2008). Our findings demonstrate that the temporal order of birth and the lobule of origin, independently of clonal relationship, specify the spatial patterning of granule neuron parallel fibers in the ML in the mammalian cerebellar cortex.

Having characterized a robust effect of birth order on granule neuron efferents, we next tested the hypothesis that birth date influences afferent connectivity and thereby impacts the diversity of granule neuron responses to sensorimotor stimuli in awake behaving mice. Analysis of movement encoding using the freewheel and motorized-wheel responsivity indices revealed that populations of early-born and late-born granule neurons both exhibited the full diversity of response selectivities to voluntary and forced locomotion (Fig. 8A). We next used linear discriminant analysis (Campbell et al., 2008) to determine whether the information about neural responsivities to the nine sensorimotor 
A
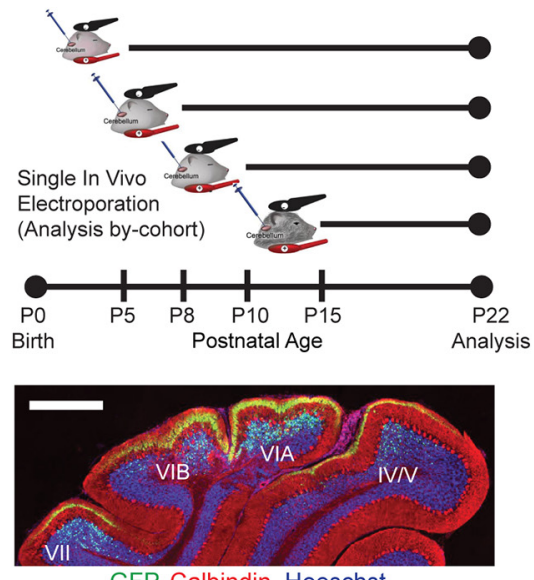

GFP, Calbindin, Hoeschst
C

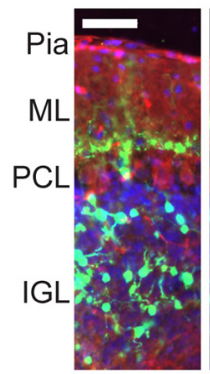

P5

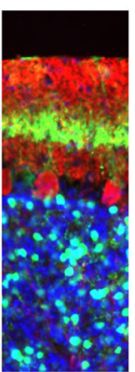

P8

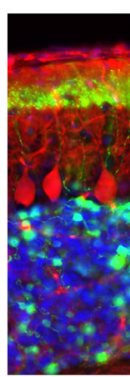

P10

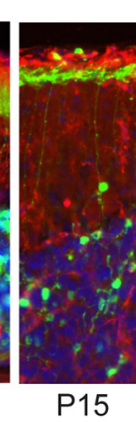

P15
Age at Electroporation (postnatal days)

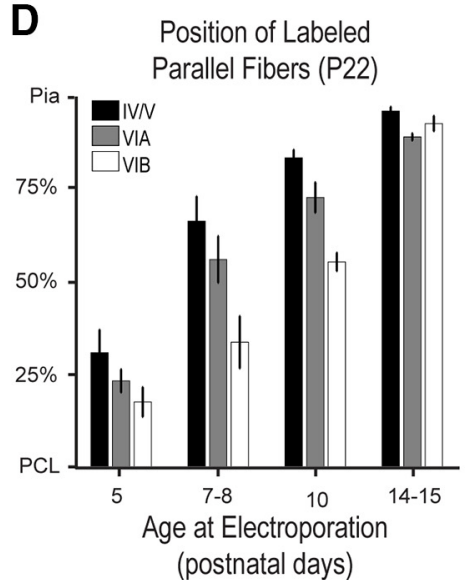

B

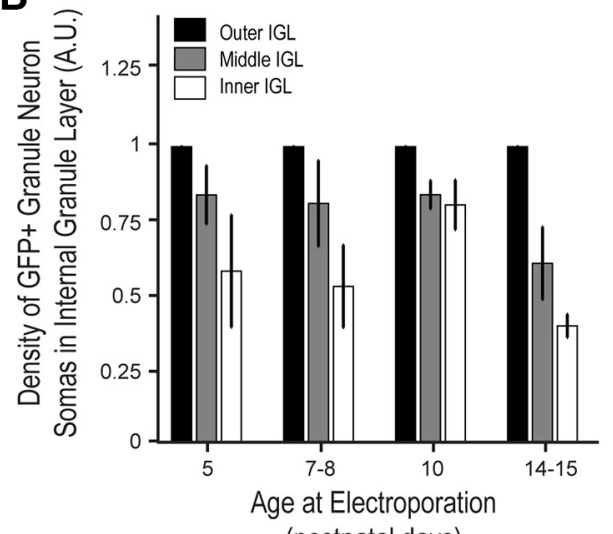

(postnatal days)
E Molecular Layer Thickness in Lobule VIA $(\mu \mathrm{m})$

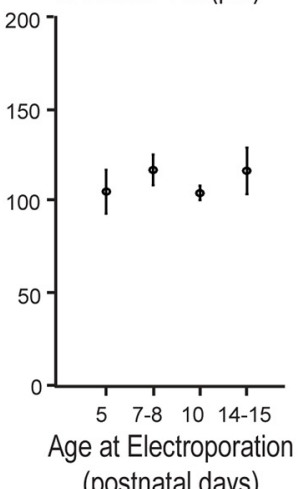

Figure 7. Parallel fibers of early-born and late-born granule neurons reside in distinct ML domains. $\boldsymbol{A}$, Top, The experimental design for evaluation of morphology of early- versus late-born granule neurons. Mice underwent in vivo electroporation between P5 and P15. At P22, the cerebellum was removed, sectioned, and subjected to immunohistochemical analyses using the GFP antibody. Bottom, Representative sagittal section at P22 following electroporation at P8 of plasmid encoding pCAG-GFP. GFP-positive granule neuron somas and parallel fibers are visible in lobules V-VII. Scale bar, $500 \mu \mathrm{m}$. B, Density of GFP-positive electroporated granule neuron somas at different depths in the IGL of lobule VI in P22 mice. Distribution of granule neuron somas in the IGL was not dependent on the age at electroporation (two-way ANOVA with no interaction of age $\times$ position, $p=0.47$; main effect of position, $F_{(2,12)}=20.401$, $p<0.001 ;$ main effect of electroporation age, $F_{(3,6)}=0.953 ; n=2$ or 3 mice per condition). C, Representative sagittal sections from lobule VI of P22 animals treated as in $A$. GFP-positive electroporated granule neuron somas reside in the IGL and their parallel fibers, running perpendicular to the plane of section, reside in the ML. PCL, Purkinje cell layer. Scale bar, $50 \mu \mathrm{m}$. D, Position of labeled parallel fibers in the molecular layer of P22 mice. Position was dependent on lobule location and age at electroporation (two-way ANOVA with interaction of age $\times$ lobule, $p=0.014 ;$ main effect of lobule, $F_{(2,18)}=27.88, p<0.001$; main effect of age, $F_{(3,9)}=22.636, p<0.001 ; n=2-5$ mice per condition). $\boldsymbol{E}$, ML thickness of P22 mice following electroporation at earlier time points. Thickness was not influenced by the age at electroporation (one-way ANOVA: $F_{(3,12)}=0.87, p=0.49 ; n=3-5$ mice per condition). All error bars denote SEM.

stimuli could be used to distinguish the two populations of earlyborn and late-born granule neurons. As a positive control, populations of granule neurons defined by the free-wheel/ motorized-wheel responsivity analysis as preferentially responsive to free-wheel (free-wheel responsivity index $>0.6$, motorized-wheel responsivity index $<0.3$ ) or motorized-wheel locomotion (free-wheel responsivity index $<0.3$, motorizedwheel responsivity index $>0.6$ ) could be reliably distinguished (90\% accuracy, $p=0.006)$ using the multikernel linear model responsivity profiles (Fig. $8 B$ ). As expected in this test case, the kernel-derived responsivities to free and forced locomotion were the most informative response variables used to distinguish the two populations. In contrast, an analogous linear discriminant analysis could not distinguish the populations of early-born and late-born granule neurons significantly better than chance based on their sensorimotor responsivities (56\% accuracy, $p=0.28$ ) (Fig. 8C). This result suggests that diverse sensorimotor stimuli are represented across populations of both early-born and lateborn granule neurons. Consequently, the diverse sensorimotor code identified at the level of cerebellar granule neurons is relayed broadly to parallel fibers throughout the molecular layer in the mammalian cerebellar cortex in vivo.

\section{Discussion}

In this study, we have elucidated response properties of granule neurons underlying the representation of sensorimotor information in the mammalian cerebellar vermis. Using a multikernel linear model to disambiguate neural responses to extrinsic cues from responses to co-occurring movements enabled quantification of the relative proportion of granule neurons responding to nine sensorimotor contexts and evaluation of the diversity of responses evoked by each stimulus. Notably, a subset of granule neurons responded differentially to locomotion on a free versus motorized wheel, and many granule neurons responded to multiple dissimilar sensorimotor stimuli. In addition, developmental in vivo electroporation studies of granule neuron responses in behaving juvenile mice revealed a diverse sensorimotor code present in both early- and late-born granule neurons whose parallel fiber axons reside in spatially segregated domains of the molecular layer. Together, our findings provide insights into the 
function and organization of vermal granule neurons in the developing mammalian cerebellum.

The convergence of diverse mossy fiber systems at the cerebellar vermis is thought to facilitate sensorimotor integration and associative learning (Stein and Glickstein, 1992; Sawtell, 2010; Huang et al., 2013; Ishikawa et al., 2015; Giovannucci et al., 2017; Knogler et al., 2017), but the extent to which stimuli of different sensory modalities activate vermal granule neurons in an untrained mouse has remained unclear. Likewise, the extent to which specific stimuli activate common or disparate populations of granule neurons has remained to be elucidated. Granule neurons in the present study were predominantly silent in the absence of stimuli, consistent with prior electrophysiological recordings (van Beugen et al., 2013; Powell et al., 2015). Locomotor stimuli activated $\sim 30 \%$ of granule neurons in lobule VIA, whereas a smaller proportion of granule neurons was activated in association with visual and auditory stimuli (Fig. 5A). The salience of locomotor signals in vermal granule neurons is supported by prior recordings in mice showing widespread increases in vermal granule neuron activity during locomotion (Ozden et al., 2012) and by recordings from granule neurons and Purkinje cells of zebrafish demonstrating broad excitation during locomotion (Knogler et al., 2017, 2019). Interestingly, we find that individual granule neurons, including those responsive to locomotion, are commonly responsive to more than one stimulus (Fig. 6), and neurons with distinct response properties are spatially intermixed (Fig. 3E). Finally, it is notable that labeling granule neurons with an activity-independent reporter revealed that nearly half of the total granule neuron population $(46 \%)$ did not respond under any of the examined conditions (Fig. 2F). Given that some vermal granule neurons may encode nonsensorimotor information, such as reward expectation (Wagner et al., 2017), and that some may change their coding properties following learning (Giovannucci et al., 2017; Wagner et al., 2019; Yamada et al., 2019), it will be of particular interest to determine how granule neurons exhibiting these different response characteristics are interrelated and whether they represent populations overlapping with or distinct from the subsets of neurons that respond to sensorimotor stimuli in untrained mice.

The variety of response time courses observed among each set of stimulus-responsive neurons (Fig. 5B-E) suggests the presence 0.28).
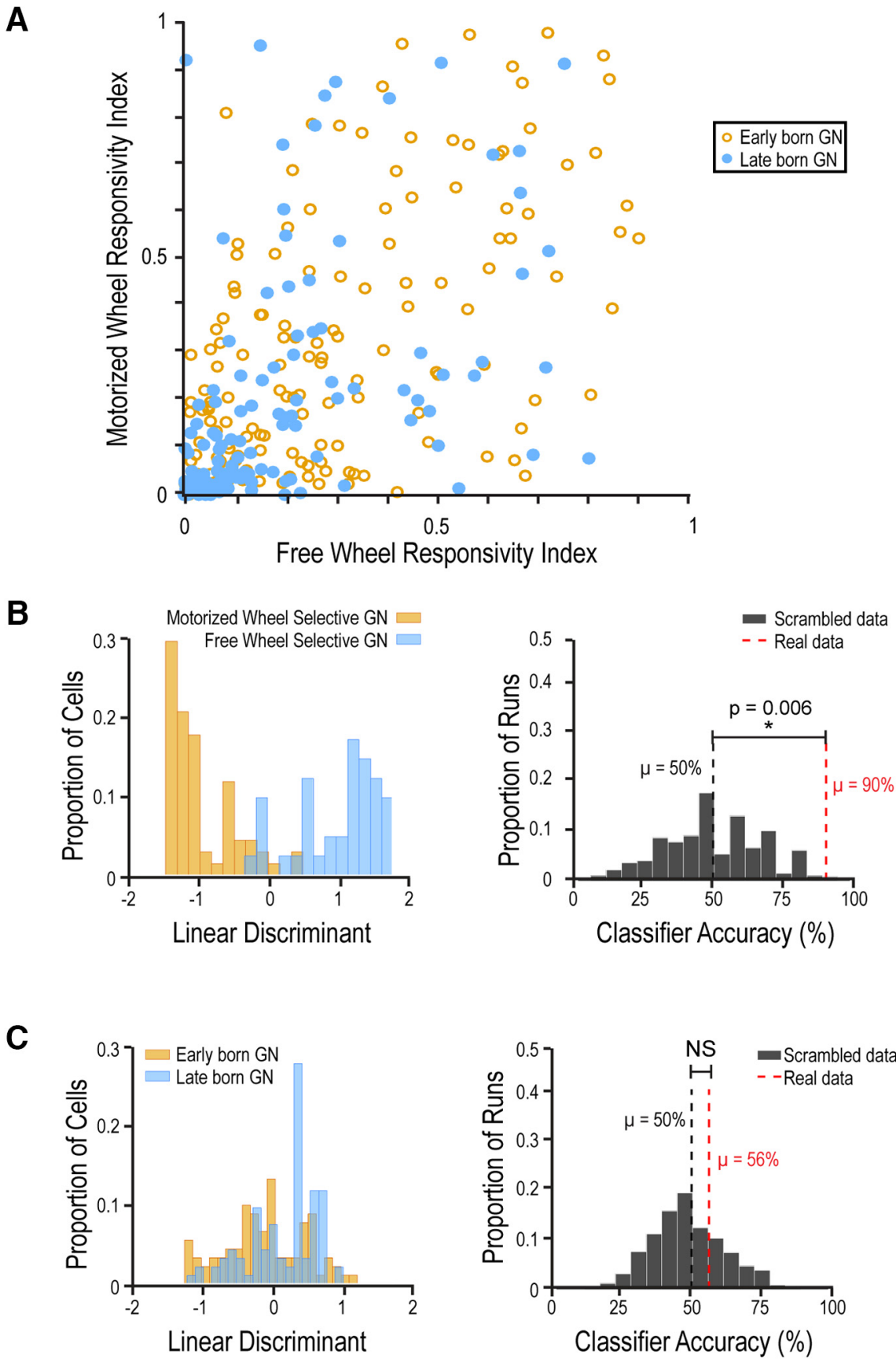

Figure 8. Both early-born and late-born granule neurons encode diverse sensorimotor information. $\boldsymbol{A}$, Free-wheel responsivity index and motorized-wheel responsivity index as in Figure 3D for early-born $(n=153)$ and late-born $(n=133)$ granule neurons (GN). $\boldsymbol{B}$, Linear discriminant analysis using the neural responsivities calculated by multikernel linear model analysis to distinguish free-wheel $(n=41)$ versus motorized-wheel $(n=68)$ selective neurons as defined by the responsivity index analysis (see Materials and Methods). Left, Linear discriminant values for the context-selective neurons. Right, The mean accuracy of the linear discriminant classifier using kernel-derived responsivities (red, $\mu=90 \%, n=5000$ bootstrapped samples) was significantly higher than the accuracy of scrambled samples (black, $\mu=50 \%, n=5000$ bootstrapped samples; $p=0.006$ ). *Statistically significant $(p<0.05)$. C, Linear discriminant analysis using the neural responsivities calculated by multikernel linear model analysis to distinguish early-born $(n=91)$ and late-born $(n=94) \mathrm{GNs}$. Left, Linear discriminant values for early-born and late-born neurons. Right, The mean accuracy of the linear discriminant classifier (red, $\mu=56 \%, n=5000$ bootstrapped samples) was not significantly higher (NS) than the accuracy of scrambled samples (black, $\mu=50 \%, n=5000$ bootstrapped samples; $p=$

of granule neuron subsets encoding distinct stimulus-associated features. Consistent with this notion, in vivo patch-clamp recordings of vermal granule neurons during locomotion indicate that the spiking of individual granule neurons can reflect different 
phases of the animal's step sequence (Powell et al., 2015), and in vivo mossy fiber recordings have identified cerebellar inputs that exhibit tonic changes in firing rate during locomotion as well as fibers that fire rhythmically (Arshavsky et al., 1972; Zangger and Schultz, 1978; Armstrong, 1986). Similarly, recordings of granule neurons in anesthetized rats during perioral air puff revealed a variety of response latencies and durations following stimulation (Duguid et al., 2015). The diversity of granule neuron responses to individual sensorimotor stimuli may also reflect the temporal diversity thought to be critical for cerebellar-dependent learning and hypothesized to arise from modulation by feedforward inhibition within the cerebellar cortex (Medina et al., 2000; Mauk and Buonomano, 2004; Kalmbach et al., 2010, 2011).

Interestingly, whereas the responses of individual neurons to locomotion and air puff stimuli were fairly consistent across repeated trials of each stimulus, the neuronal responses to visual and auditory stimuli showed less trial-to-trial consistency (Fig. $5 A$ ). As opposed to reflecting a noisy encoding of the animal's stimulus perception, the neural variability may arise from behavioral variability and reflect faithful encoding of features of the animal's response (e.g., twitch, eyeblink, or whisking) that are inconsistently evoked by stimulus presentation (Lisberger and Medina, 2015). Together, our data provide direct quantification of the relative activation of vermal granule neurons in response to sensorimotor stimuli and offer insights into the temporal variety of neural responses associated with each stimulus. Future studies recording granule neuron activity in conjunction with more detailed video analyses and EMG monitoring will help further delineate whether and how specific behavioral parameters relate to the observed responses. Notably, the multikernel linear model approach used in this study may aid interpretation of such studies by allowing the proportion of calcium activity explained by multiple co-occurring behavioral and external events to be disentangled.

The identification of cerebellar granule neurons that respond differentially to locomotion in a free-wheel versus motorizedwheel context (Figs. 3D, 4E) bears significant implications for information processing in the cerebellum. Distinguishing active self-generated movements from passive externally applied ones is thought to be critical for accurate perception and motor control (Von Holst and Mittelstaedt, 1950; Angelaki and Cullen, 2008), and the cerebellum may be a key site for this computation (Roy and Cullen, 2001, 2004; Brooks and Cullen, 2013). Vermalprojecting mossy fibers selectively responsive to active versus passive limb movements have previously been identified (van Kan et al., 1993), but monitoring for such responses from granule neurons in vivo has not been feasible until recently. Accordingly, an intriguing possibility is that some granule neurons selectively responsive to the motorized wheel encode passive aspects of movement, as the mouse's limbs are displaced by external forces not present during free-wheel locomotion. Conversely, selective granule neuron responses to the free-wheel locomotion may reflect an efference copy signal, as the mouse voluntarily initiates a motor command. Granule neurons active under both free and forced locomotion may convey signals present in both contexts, including proprioceptive, active movement, or efference copy information. The prevalence and diversity of these locomotor-related signals add to the growing body of evidence of the importance of motor signals in granule neurons across diverse contexts (Albergaria et al., 2018; Khilkevich et al., 2018; Knogler et al., 2019) and suggest that the information encoded in lobule VI vermis is poised to impact locomotor coordination and adaptation.
To probe the factors regulating establishment of the sensorimotor code during development, we used in vivo electroporation and investigated cohorts of hundreds of nonclonal granule neurons that share a similar birth date. These studies revealed that parallel fiber axons are positioned in the molecular layer in a birth date- and lobule-specific fashion, and suggest that the information carried by the earliest-born granule neurons may be preferentially transmitted to the proximal Purkinje cell dendritic arbor and to basket cells that reside in the deep molecular layer (Fig. 7). By contrast, late-born granule neurons transmit their information to the distal dendritic arbors and may communicate preferentially with stellate cells in the superficial molecular layer. Although the location of spike generation on the Purkinje cell dendritic tree does not significantly impact somatic firing (Rancz and Häusser, 2010), molecular layer interneurons exhibit spatially structured connectivity patterns (Rieubland et al., 2014), suggesting that early- and late-born granule neurons might differentially affect molecular layer processing. In addition, functionally similar parallel fiber inputs exhibit spatial clustering, and birth order-derived functional differences between granule neurons have been hypothesized as one possible mechanism for this arrangement (Wilms and Häusser, 2015). We provide the first comparison of functional properties of early- versus late-born granule neurons in the mammalian cerebellum, demonstrating that a diverse sensorimotor code is processed in both early- and late-born granule neurons and suggesting that this information is relayed broadly across the molecular layer (Fig. 8). These results suggest that the birth order of granule neurons influences the structural assembly of parallel fiber connections with their efferent synaptic partners but does not have an obvious relationship to representations of incoming sensorimotor information. It will be interesting in future studies to evaluate whether mossy fibers conveying nonsensorimotor information (e.g., cognitive, limbic) connect differentially with early- versus late-born granule neurons and to further probe the impact of early- versus late-born granule neurons on information processing in the molecular layer.

In conclusion, these results define key properties of the coding and organization of sensorimotor information in granule neurons of the cerebellar vermis, including the rate and type of responses evoked by visual, auditory, somatosensory, and locomotor stimuli. The in vivo response properties reported here provide insight into the organization of sensorimotor information arriving to the cerebellar vermis and pave the way for future studies examining how the cerebellum uses this information for associative learning and motor control, including postural control and locomotion.

\section{References}

Aitkin LM, Boyd J (1975) Responses of single units in cerebellar vermis of the cat to monaural and binaural stimuli. J Neurophysiol 38:418-429.

Albergaria C, Silva NT, Pritchett DL, Carey MR (2018) Locomotor activity modulates associative learning in mouse cerebellum. Nat Neurosci 21: $725-735$.

Altman J (1972) Postnatal development of the cerebellar cortex in the rat: III. Maturation of the components of the granular layer. J Comp Neurol 145:465-513.

Altman J, Bayer SA (1987) Development of the precerebellar nuclei in the rat: IV. The anterior precerebellar extramural migratory stream and the nucleus reticularis tegmenti pontis and the basal pontine gray. J Comp Neurol 257:513-528.

Altman J, Bayer SA (1997) Development of the cerebellar system: in relation to its evolution, structure, and functions. Boca Raton, FL: CRC.

Angelaki DE, Cullen KE (2008) Vestibular system: the many facets of a multimodal sense. Annu Rev Neurosci 31:125-150. 
Apps R, Hawkes R, Aoki S, Bengtsson F, Brown AM, Chen G, Ebner TJ, Isope P, Jörntell H, Lackey EP, Lawrenson C, Lumb B, Schonewille M, Sillitoe RV, Spaeth L, Sugihara I, Valera A, Voogd J, Wylie DR, Ruigrok TJ (2018) Cerebellar modules and their role as operational cerebellar processing units: a consensus paper. Cerebellum 5:654-682.

Arenz A, Bracey EF, Margrie TW (2009) Sensory representations in cerebellar granule cells. Curr Opin Neurobiol 19:445-451.

Armstrong DM (1986) Supraspinal contributions to the initiation and control of locomotion in the cat. Prog Neurobiol 26:273-361.

Armstrong DM (1988) The supraspinal control of mammalian locomotion. J Physiol 405:1-37.

Arshavsky YI, Berkinblit MB, Fukson OI, Gelfand IM, Orlovsky GN (1972) Recordings of neurons of the dorsal spinocerebellar tract during evoked locomotion. Brain Res 43:272-275.

Ashwell KW, Zhang L (1992) Ontogeny of afferents to the fetal rat cerebellum. Acta Anat (Basel) 145:17-23.

Bastian AJ, Mink JW, Kaufman BA, Thach WT (1998) Posterior vermal split syndrome. Ann Neurol 44:601-610.

Bengtsson F, Jörntell H (2009) Sensory transmission in cerebellar granule cells relies on similarly coded mossy fiber inputs. Proc Natl Acad Sci U S A 106:2389-2394.

Billings G, Piasini E, Lorincz A, Nusser Z, Silver RA (2014) Network structure within the cerebellar input layer enables lossless sparse encoding. Neuron 83:960-974.

Bosco G, Eian J, Poppele RE (2005) Kinematic and non-kinematic signals transmitted to the cat cerebellum during passive treadmill stepping. Exp Brain Res 167:394-403.

Brooks JX, Cullen KE (2013) The primate cerebellum selectively encodes unexpected self-motion. Curr Biol 23:947-955.

Campbell RA, King AJ, Nodal FR, Schnupp JW, Carlile S, Doubell TP (2008) Virtual adult ears reveal the roles of acoustical factors and experience in auditory space map development. J Neurosci 28:11557-11570.

Chabrol FP, Arenz A, Wiechert MT, Margrie TW, DiGregorio DA (2015) Synaptic diversity enables temporal coding of coincident multisensory inputs in single neurons. Nat Neurosci 18:718-727.

Chen TW, Wardill TJ, Sun Y, Pulver SR, Renninger SL, Baohan A, Schreiter ER, Kerr RA, Orger MB, Jayaraman V, Looger LL, Svoboda K, Kim DS (2013) Ultrasensitive fluorescent proteins for imaging neuronal activity. Nature 499:295-300.

Coffman KA, Dum RP, Strick PL (2011) Cerebellar vermis is a target of projections from the motor areas in the cerebral cortex. Proc Natl Acad Sci U S A 108:16068-16073.

Dayan P, Abbott LF (2005) Theoretical neuroscience: computational and mathematical modeling of neural systems. Cambridge: Massachusetts Institute of Technology.

Dombeck D, Tank D (2014) Two-photon imaging of neural activity in awake mobile mice. Cold Spring Harb Protoc 7:726-736.

Duguid I, Branco T, Chadderton P, Arlt C, Powell K, Häusser M (2015) Control of cerebellar granule cell output by sensory-evoked Golgi cell inhibition. Proc Natl Acad Sci U S A 112:13099-13104.

Ekerot CF, Jörntell H (2008) Synaptic integration in cerebellar granule cells. Cerebellum 7:539-541.

Espinosa JS, Luo L (2008) Timing neurogenesis and differentiation: insights from quantitative clonal analyses of cerebellar granule cells. J Neurosci 28:2301-2312.

Fünfschilling U, Reichardt LF (2002) Cre-mediated recombination in rhombic lip derivatives. Genesis 33:160-169.

Gebre SA, Reeber SL, Sillitoe RV (2012) Parasagittal compartmentation of cerebellar mossy fibers as revealed by the patterned expression of vesicular glutamate transporters VGLUT1 and VGLUT2. Brain Struct Funct 217: $165-180$.

Gilmer JI, Person AL (2017) Morphological constraints on cerebellar granule cell combinatorial diversity. J Neurosci 37:12153-12166.

Giovannucci A, Badura A, Deverett B, Najafi F, Pereira TD, Gao Z, Ozden I, Kloth AD, Pnevmatikakis E, Paninski L, De Zeeuw CI, Medina JF, Wang SS (2017) Cerebellar granule cells acquire a widespread predictive feedback signal during motor learning. Nat Neurosci 20:727-734.

Glickstein M (1997) Mossy-fibre sensory input to the cerebellum. In: The cerebellum: from structure to control (De Zeeuw CI, Strata P, Voogd J, eds), pp 251-259. Amsterdam: Elsevier.

Heiney SA, Wohl MP, Chettih SN, Ruffolo LI, Medina JF (2014) Cerebellar- dependent expression of motor learning during eyeblink conditioning in head-fixed mice. J Neurosci 34:14845-14853.

Holmes G (1922) Clinical symptoms of cerebellar disease: Lecture III. Lancet 200:59-65.

Huang CC, Sugino K, Shima Y, Guo C, Bai S, Mensh BD, Nelson SB, Hantman AW (2013) Convergence of pontine and proprioceptive streams onto multimodal cerebellar granule cells. Elife 2:e00400.

Huang CM, Liu G,Huang R (1982) Projections from the cochlear nucleus to the cerebellum. Brain Res 244:1-8.

Huynh MA, Ikeuchi Y, Netherton S, de la Torre-Ubieta L, Kanadia R, Stegmüller J, Cepko C, Bonni S, Bonni A (2011) An isoform-specific SnoN1FOXO1 repressor complex controls neuronal morphogenesis and positioning in the mammalian brain. Neuron 69:930-944.

Ishikawa T, Shimuta M, Häusser M (2015) Multimodal sensory integration in single cerebellar granule cells in vivo. Elife 4:e12916.

Ji Z, Hawkes R (1994) Topography of Purkinje cell compartments and mossy fiber terminal fields in lobules II and III of the rat cerebellar cortex: spinocerebellar and cuneocerebellar projections. Neuroscience 61:935954.

Jörntell H, Ekerot CF (2006) Properties of somatosensory synaptic integration in cerebellar granule cells in vivo. J Neurosci 26:11786-11797.

Kalinovsky A, Boukhtouche F, Blazeski R, Bornmann C, Suzuki N, Mason CA, Scheiffele P (2011) Development of axon-target specificity of ponto-cerebellar afferents. PLoS Biol. 9:e1001013.

Kalmbach BE, Ohyama T, Mauk MD (2010) Temporal patterns of inputs to cerebellum necessary and sufficient for trace eyelid conditioning. J Neurophysiol 104:627-640.

Kalmbach BE, Voicu H, Ohyama T, Mauk MD (2011) A subtraction mechanism of temporal coding in cerebellar cortex. J Neurosci 31:2025-2034.

Kelly RM, Strick PL (2003) Cerebellar loops with motor cortex and prefrontal cortex of a nonhuman primate. J Neurosci 23:8432-8444.

Khilkevich A, Zambrano J, Richards MM, Mauk MD (2018) Cerebellar implementation of movement sequences through feedback. Elife 7:e37443.

Knogler LD, Markov DA, Dragomir EI, Stih V, Portugues R (2017) Sensorimotor representations in cerebellar granule cells in larval zebrafish are dense, spatially organized, and non-temporally patterned. Curr Biol 27: $1288-1302$.

Knogler LD, Kist AM, Portugues R (2019) Motor context dominates output from Purkinje cell functional regions during reflexive visuomotor behaviours. Elife 8:e42138.

Lisberger SG, Medina JF (2015) How and why neural and motor variation are related. Curr Opin Neurobiol 33:110-116.

Maruyama R, Maeda K, Moroda H, Kato I, Inoue M, Miyakawa H, Aonishi T (2014) Detecting cells using non-negative matrix factorization on calcium imaging data. Neural Netw 55:11-19.

Matsushita M, Ikeda M (1980) Spinocerebellar projections to the vermis of the posterior lobe and the paramedian lobule in the cat, as studied by retrograde transport of horseradish peroxidase. J Comp Neurol 192:143162.

Matsushita M, Hosoya Y, Ikeda M (1979) Anatomical organization of the spinocerebellar system in the cat, as studied by retrograde transport of horseradish peroxidase. J Comp Neurol 184:81-106.

Mauk MD, Buonomano DV (2004) The neural basis of temporal processing. Annu Rev Neurosci 27:307-340.

Medina JF, Garcia KS, Nores WL, Taylor NM, Mauk MD (2000) Timing mechanisms in the cerebellum: testing predictions of a large-scale computer simulation. J Neurosci 20:5516-5525.

Orlovsky GN, Deliagina TG, Grillner S (1999) Neuronal control of locomotion. Oxford: Oxford Neuroscience Series.

Ozden I, Dombeck DA, Hoogland TM, Tank DW, Wang SS (2012) Widespread state-dependent shifts in cerebellar activity in locomoting mice. PLoS One 7:e42650.

Päällysaho J, Sugita S, Noda H (1991) Brainstem mossy fiber projections of lobules VIa, VIb,c, VII and VIII of the cerebellar vermis in the rat. Neurosci Res 12:217-231.

Peters AJ, Chen SX, Komiyama T (2014) Emergence of reproducible spatiotemporal activity during motor learning. Nature 510:263-267.

Pnevmatikakis EA, Giovannucci A (2017) NoRMCorre: an online algorithm for piecewise rigid motion correction of calcium imaging data. J Neurosci Methods 291:83-94.

Pnevmatikakis EA, Soudry D, Gao Y, Machado TA, Merel J, Pfau D, Reardon T, Mu Y, Lacefield C, Yang W, Ahrens M, Bruno R, Jessell TM, Peterka 
DS, Yuste R, Paninski L (2016) Simultaneous denoising, deconvolution, and demixing of calcium imaging data. Neuron 89:285-299.

Powell K, Mathy A, Duguid I, Häusser M (2015) Synaptic representation of locomotion in single cerebellar granule cells. Elife 4:07290.

Ramon y Cajal, S (1911) Histologie du System Nerveux de l'Homme et des Vertebres. Paris: A. Malonine.

Rancz EA, Häusser M (2010) Dendritic spikes mediate negative synaptic gain control in cerebellar Purkinje cells. Proc Natl Acad Sci U S A 107:22284-22289.

Rieubland S, Roth A, Häusser M (2014) Structured connectivity in cerebellar inhibitory networks. Neuron 81:913-929.

Roy JE, Cullen KE (2001) Selective processing of vestibular reafference during self-generated head motion. J Neurosci 21:2131-2142.

Roy JE, Cullen KE (2004) Dissociating self-generated from passively applied head motion: neural mechanisms in the vestibular nuclei. J Neurosci 24:2102-2111.

Sawtell NB (2010) Multimodal integration in granule cells as a basis for associative plasticity and sensory prediction in a cerebellum-like circuit. Neuron 66:573-584.

Shik ML, Orlovsky GN (1976) Neurophysiology of locomotor automatism. Physiol Rev 56:465-501.

Simoncelli EP, Paninski L, Pillow JW, Schwartz O (2004) Characterization of neural responses with stochastic stimuli. In: The new cognitive neurosciences, pp 327-338. Cambridge: Massachusetts Institute of Technology.

Snider RS, Stowell A (1944) Receiving areas of the tactile, auditory, and visual systems in the cerebellum. J Neurophysiol 7:331-358.

Stein JF, Glickstein M (1992) Role of the cerebellum in visual guidance of movement. Physiol Rev 72:967-1017.

Stoodley CJ, Schmahmann JD (2009) Neuroimage functional topography in the human cerebellum: a meta-analysis of neuroimaging studies. Neuroimage 44:489-501.

Thach WT, Bastian AJ (2004) Role of the cerebellum in the control and adaptation of gait in health and disease. Prog Brain Res 143:353-366.

Tian L, Hires SA, Mao T, Huber D, Chiappe ME, Chalasani SH, Petreanu L, Akerboom J, McKinney SA, Schreiter ER, Bargmann CI, Jayaraman V, Svoboda K, Looger LL (2009) Imaging neural activity in worms, flies and mice with improved GCaMP calcium indicators. Nat Methods 6:875-881.

Valnegri P, Huang J, Yamada T, Yang Y, Mejia LA, Cho HY, Oldenborg A, Bonni A (2017) RNF8/UBC13 ubiquitin signaling suppresses synapse formation in the mammalian brain. Nat Commun 8:1271.

van Beugen BJ, Gao Z, Boele HJ, Hoebeek F, De Zeeuw CI (2013) High frequency burst firing of granule cells ensures transmission at the parallel fiber to Purkinje cell synapse at the cost of temporal coding. Front Neural Circuits 7:95.

van Kan PL, Gibson AR, Houk JC (1993) Movement-related inputs to intermediate cerebellum of the monkey. J Neurophysiol 69:74-94.

Vinueza Veloz MF, Zhou K, Bosman LW, Potters JW, Negrello M, Seepers RM, Strydis C, Koekkoek SK, De Zeeuw CI (2015) Cerebellar control of gait and interlimb coordination. Brain Struct Funct 220:3513-3536.

Von Holst E, Mittelstaedt H (1950) Das reafferenz princip. Naturwissenschften 464:476.

Wagner MJ, Kim TH, Savall J, Schnitzer MJ, Luo L (2017) Cerebellar granule cells encode the expectation of reward. Nature 544:96-100.

Wagner MJ, Kim TH, Kadmon J, Nguyen ND, Ganguli S, Schnitzer MJ, Luo L (2019) Shared cortex-cerebellum dynamics in the execution and learning of a motor task. Cell 177:669-682.e24.

Wiesendanger R, Wiesendanger M (1982) The corticopontine system in the rat: I. Mapping of corticopontine neurons. J Comp Neurol 208:215-226.

Wilms CD, Häusser M (2015) Reading out a spatiotemporal population code by imaging neighbouring parallel fibre axons in vivo. Nat Commun 6:6464.

Witter L, De Zeeuw CI (2015) In vivo differences in inputs and spiking between neurons in lobules VI/VII of neocerebellum and lobule X of archaeocerebellum. Cerebellum 14:506-515

Yamada T, Yang Y, Hemberg M, Yoshida T, Cho HY, Murphy JP, Fioravante D, Regehr WG, Gygi SP, Georgopoulos K, Bonni A (2014) Promoter decommissioning by the NuRD chromatin remodeling complex triggers synaptic connectivity in the mammalian brain. Neuron 83:122-134.

Yamada T, Yang Y, Valnegri P, Juric I, Abnousi A, Markwalter KH, Guthrie AN, Godec A, Oldenborg A, Hu M, Holy TE, Bonni A (2019) Sensory experience remodels genome architecture in neural circuit to drive motor learning. Nature 569:708-713.

Yang Y, Yamada T, Hill KK, Hemberg M, Reddy NC, Cho HY, Guthrie AN, Oldenborg A, Heiney SA, Ohmae S, Medina JF, Holy TE, Bonni A (2016) Chromatin remodeling inactivates activity genes and regulates neural coding. Science 353:300-305.

Zangger P, Schultz W (1978) The activity of cells of nucleus reticularis tegmenti pontis during spontaneous locomotion in the decorticate cat. Neurosci Lett 7:95-99.

Zhang Q, Mason CA (1998) Developmental regulation of mossy fiber afferent interactions with target granule cells. Dev Biol 195:75-87.

Zong H, Espinosa JS, Su HH, Muzumdar MD, Luo L (2005) Mosaic analysis with double markers in mice. Cell 121:479-492. 